\title{
Asymptotic Equivalence Between the Unconditional Maximum Likelihood and the Square-Law Nonlinearity Symbol Timing Estimation
}

\author{
José A. López-Salcedo, Student Member, IEEE, and Gregori Vázquez, Senior Member, IEEE
}

\begin{abstract}
This paper provides a systematic approach to the problem of nondata aided symbol-timing estimation for linear modulations. The study is performed under the unconditional maximum likelihood framework where the carrier-frequency error is included as a nuisance parameter in the mathematical derivation. The second-order moments of the received signal are found to be the sufficient statistics for the problem at hand and they allow the provision of a robust performance in the presence of a carrier-frequency error uncertainty. We particularly focus on the exploitation of the cyclostationary property of linear modulations. This enables us to derive simple and closed-form symbol-timing estimators which are found to be based on the well-known square timing recovery method by Oerder and Meyr. Finally, we generalize the OM method to the case of linear modulations with offset formats. In this case, the square-law nonlinearity is found to provide not only the symbol-timing but also the carrier-phase error.
\end{abstract}

Index Terms-Cyclostationarity, nondata aided, phase estimation, second-order statistics, synchronization, timing.

\section{INTRODUCTION}

$\mathbf{T}$ HE problem of symbol-timing estimation is one of the basic tasks of any digital receiver. In this sense, a proper alignment between the incoming signal and the sampling time instants is required for the absence of intersymbol interference (ISI) and optimum symbol detection at the matched filter output. However, working scenarios with significant low signal-to-noise ratio (SNR) are usually encountered. Under these circumstances, nondata-aided (NDA) techniques appear as the most dynamic and efficient way to perform the parameter estimation [1]. These techniques have received significant attention in the recent years, but especially those which rely on exploiting the cyclostationarity of linear modulations [2].

For the particular case of NDA timing estimation, a widespread procedure is based on passing the received signal into

\footnotetext{
Manuscript received February 9, 2004; revised February 16, 2005. This work was financed by the Spanish/Catalan Science and Technology Commissions and FEDER funds from the European Commission: TIC2001-2356, TIC2003-05482, TEC2004-04526, and 2001SGR-00268. The material in this paper was presented in part at the IEEE International Conference on Communications (ICC), Anchorage, AK, USA, May 2003 and at the IEEE International Conference on Speech, Acoustics and Signal Processing (ICASSP), Montreal, QC, Canada, May 2004. The associate editor coordinating the review of this paper and approving it for publication was Prof. Abdelhak M. Zoubir.

The authors are with the Department of Signal Theory and Communications, Technical University of Catalonia (UPC), Mòdul D5-117, Campus Nord UPC, 08034 Barcelona, Spain (e-mail: jlopez@gps.tsc.upc.edu; gregori@ gps.tsc.upc.edu).

Digital Object Identifier 10.1109/TSP.2005.859245
}

a nonlinear device and feeding the output to a narrow-band phase-locked loop (PLL) for obtaining the timing phase error [3], [4]. Several contributions have further analyzed this procedure by studying the impact of the nonlinearity to be applied or the optimal pulse shape filter for achieving the best performance. See for example some historical references such as [5]-[8]. However, it is interesting to note that among the wide range of NDA timing synchronizers, the well-known Oerder and Meyr (OM) algorithm [9] is probably the mostly adopted in practical applications. This technique is based on applying a second-order nonlinearity to the received signal and extracting the timing information from the spectral line that has been generated at the symbol rate. Although this technique was initially proposed under heuristic reasonings, the robust performance of the OM algorithm in low SNR scenarios has been recently related to the one provided by maximum likelihood (ML) estimation. Motivated by this fact, the authors in [10] show that many of the existing timing estimators based on the second-order cyclostationary statistics (CSS) may indeed be asymptotically interpreted as ML estimators.

Following on this topic, this paper concentrates on the general formulation of the timing estimation problem under the Stochastic or Unconditional Maximum Likelihood (UML) approach. Particularly, this timing estimation is performed among a set of synchronization parameters that also include the carrierphase and the carrier-frequency error as the nuisance parameters. This paper provides a closed-form expression for the general timing estimation under an unknown and random carrierfrequency error whose statistical behavior is characterized by a given probability density function. For the case of maximum carrier-frequency uncertainty, the derived timing estimator simplifies to the OM algorithm without receiving prefilter, as already pointed out by [1]. However, for the case of perfect carrier frequency acquisition, we find that the derived timing estimator also simplifies to the OM algorithm, but the receiving prefilter is now given by a filter matched to the transmitted pulse shape. Hence, the popular OM algorithm is found to be derived from an analytic and systematic ML formulation, which finally establishes a clear link between this technique and ML estimation.

The main part of this paper is focused on the asymptotic performance for the case of linear modulations with nonoffset format. Nevertheless, the systematic formulation of the ML cost function also allows us to easily extend the derived results to the case of offset formats (i.e., staggered modulations). In this case, a joint phase and timing estimator can be derived which is also found to be based on the simple OM principle. Linear 
modulations with offset format belong to the class of bandwidth efficient modulations, providing a good ratio between the transmitted signaling rate with respect to the transmission bandwidth. The main difference with respect to nonoffset modulations is that the in-phase and the quadrature data streams are not aligned in time. Thus, smoother phase transitions are achieved. The importance of this time offset is that it solves most of the problems encountered by linear modulations when passing through nonlinear devices such as High Power Amplifiers (see [11] and the references therein).

The structure of the paper is as follows. Section II provides the signal model and some important assumptions for the nonoffset and offset modulation formats. Next, Section III presents the UML timing estimation framework. Therein, the CSS of the received signal are exploited for obtaining a closed-form timing estimator. Later on, this closed-form timing estimator is particularized for an asymptotically large observation interval in Section IV. The results are extended to the case of offset modulations in Section V and simulation results are presented in Section VI. Finally, conclusions are drawn in Section VII.

\section{Signal Model AND PRELiminary Considerations}

The mathematical framework for the UML estimation is introduced in the present paper for the case of linear modulations with nonoffset format. Later on, and taking into consideration the results provided up to that point, a generalization is presented to the case of linear modulations with offset format. For this reason, both signal models are herein introduced.

\section{A. Signal Model for Linear Modulations With Nonoffset Format}

We focus on the following continuous-time signal model for the baseband received signal ${ }^{1}$

$$
r_{c}(t)=\sum_{n=-\infty}^{\infty} x_{n} g_{c}\left(t-n T-\tau_{c}\right) e^{j\left(2 \pi f_{\varepsilon} t+\theta_{0}\right)}+w_{c}(t)
$$

where $\left\{x_{n}\right\}$ stands for the independent identically distributed (i.i.d.) transmitted symbols, $g_{c}(t)$ is the real-symmetric transmitted pulse shape filter and $w_{c}(t)$ is the complex additive white Gaussian noise (AWGN) with two-sided power spectral density $S_{w}(f)=2 N_{o} \mathrm{~W} / \mathrm{Hz}$. The symbol period is denoted by $T$, and the set of unknown nuisance parameters includes the continuous-time symbol-timing error $\tau_{c}$, the carrier-frequency error $f_{\varepsilon}$ and the carrier-phase error $\theta_{0}$. The continuous-time received signal $r_{c}(t)$ is passed through an ideal antialiasing filter of bandwidth $f_{s} / 2$ and sampled at a rate $f_{s}=1 / T_{s}$. The sampling period is equal to $T_{s}=T / N_{s s}$, with $N_{s s}$ the number of samples per symbol.

In this way, the following discrete-time signal model is obtained

$$
r(k)=\sum_{n=-\infty}^{\infty} x_{n} g\left(k-n N_{s s}-\tau\right) e^{j\left(\left(2 \pi / N_{s s}\right) \nu k+\theta_{0}\right)}+w(k)
$$

${ }^{1}$ The subscript $(\cdot)_{c}$ denotes continuous-time. with $r(k) \doteq r_{c}\left(k T_{s}\right), g(k) \doteq g_{c}\left(k T_{s}\right)$ and $w(k) \doteq w_{c}\left(k T_{s}\right)$. The discrete-time symbol-timing error $\tau \doteq \tau_{c} / T_{s}$ is constrained within the interval $\left[-N_{s s} / 2,+N_{s s} / 2\right)$, and the carrier-frequency error has been normalized to the symbol rate, that is $\nu \doteq f_{\varepsilon} T$ with $\nu \in\left[-N_{s s} / 2,+N_{s s} / 2\right)$. In order to facilitate the mathematical treatment, the discrete-time signal model in (2) is henceforth represented in matrix notation. Thus, taking into consideration a symmetric observation interval comprising $L \doteq 2 K+1$ transmitted symbols, the vector $\mathbf{r}$ consists of $N \doteq 2 M+1$ received samples according to the following signal model:

$$
\mathbf{r}=\mathbf{A}(\boldsymbol{\Theta}) \mathbf{x}+\mathbf{w}
$$

where

$$
\begin{aligned}
\mathbf{r} \doteq & {[r(-M), r(-M+1), \ldots, r(M)]^{T} } \\
\mathbf{A}(\boldsymbol{\Theta}) \doteq & {\left[\mathbf{a}_{-K}(\boldsymbol{\Theta}), \mathbf{a}_{-K+1}(\boldsymbol{\Theta}), \ldots, \mathbf{a}_{K}(\boldsymbol{\Theta})\right] } \\
\mathbf{a}_{n}(\boldsymbol{\Theta}) \doteq & {\left[g\left(-n N_{s s}-M-\tau\right) e^{-j\left(2 \pi / N_{s s}\right) M \nu}\right.} \\
& g\left(-n N_{s s}-(M-1)-\tau\right) e^{-j\left(2 \pi / N_{s s}\right)(M-1) \nu} \\
& \left.\ldots, g\left(-n N_{s s}+M-\tau\right) e^{j\left(2 \pi / N_{s s}\right) M \nu}\right]^{T} e^{j \theta_{0}} \\
\mathbf{x} \doteq & {\left[x_{-K}, x_{-K+1}, \ldots, x_{K}\right]^{T} } \\
\mathbf{w} \doteq & {[w(-M), w(-M+1), \ldots, w(M)]^{T} }
\end{aligned}
$$

In the sequel, $(\cdot)^{T}$ and $(\cdot)^{H}$ stand for the transpose and the conjugate transpose operators respectively. An important remark is that the linear transfer matrix $\mathbf{A}(\boldsymbol{\Theta})$ depends on the vector of unknown synchronization parameters $\boldsymbol{\Theta}=\left[\tau, \theta_{0}, \nu\right]^{T}$. However, and for the sake of simplicity, this dependence is often omitted in the notation.

For the case of linear modulations with nonoffset format, the following assumptions are considered.

AS1a) The transmitted symbols are i.i.d, zero mean, unit variance, $\mathrm{E}\left[\left|x_{n}\right|^{2}\right]=1$, and can be selected from either a circular or a noncircular constellation.

AS2a) The pulse shape filter $g(k)$ is the discrete-time version of a bandlimited pulse shape. Typically, this is a square root raised cosine (SQRRC) pulse whose bandwidth is limited to $|f| \leq(1+\beta) / 2 T$, with $\beta$ the roll-off factor, $\beta \in[0,1]$.

AS3a) The carrier-phase offset $\theta_{0}$ is assumed to be a uniformly distributed random variable within $[-\pi, \pi)$.

AS4a) The normalized carrier-frequency error $\nu$ is assumed to be an unknown random parameter constrained within the interval $\nu \in\left[-\Delta_{\nu} / 2,+\Delta_{\nu} / 2\right)$ with a probability density function (pdf) given by $f_{\nu}(\nu)$.

AS5a) The observation interval is asymptotically large, that is, $(K \rightarrow \infty)$ and $(M \rightarrow \infty)$.

\section{B. Signal Model for Linear Modulations With Offset Format}

The staggered modulations considered in this paper assume a time offset between the in-phase and the quadrature data streams equal to half the symbol period. Following the same notation as 
in Section II-A, the discrete-time signal model for the baseband received staggered signal is given by

$$
\begin{aligned}
r(k)= & e^{j\left(\left(2 \pi / N_{s s}\right) \nu k+\theta_{0}\right)} \\
& \cdot \sum_{n=-\infty}^{\infty}\left[x_{n}^{\mathcal{R}} g\left(k-n N_{s s}-\tau\right)+j x_{n}^{\mathcal{I}} g\left(k-n N_{s s}-\frac{N_{s s}}{2}-\tau\right)\right] \\
& +w(k)
\end{aligned}
$$

where an even number of samples per symbol $N_{s s}$ is considered and $\left\{x_{n}^{\mathcal{R}}, x_{n}^{\mathcal{I}}\right\}$ stand for the in-phase and the staggered quadrature symbols, respectively.

The signal model in (9) shares almost all the signal parameters already defined in Section II-A except for $\left\{x_{n}^{\mathcal{R}}, x_{n}^{\mathcal{I}}\right\}$ which are now defined as the transmitted symbols corresponding to the in-phase and the staggered quadrature component, respectively. As for Section II-A, we can also define the signal model in matrix notation as

$$
\mathbf{r}=\mathbf{A}(\boldsymbol{\Theta}) \mathbf{x}_{\mathcal{R}}+j \mathbf{J} \mathbf{A}(\boldsymbol{\Theta}) \mathbf{x}_{\mathcal{I}}+\mathbf{w}
$$

where $\left\{\mathbf{x}_{\mathcal{R}}, \mathbf{x}_{\mathcal{I}}\right\}$, and the $(p, q)$ entries of the matrix $\mathbf{J}$ are now given by

$$
\begin{aligned}
\mathbf{x}_{\mathcal{R}} & \doteq\left[x_{-K}^{\mathcal{R}}, x_{-K+1}^{\mathcal{R}}, \ldots, x_{K}^{\mathcal{R}}\right]^{T} \\
\mathbf{x}_{\mathcal{I}} & \doteq\left[x_{-K}^{\mathcal{I}}, x_{-K+1}^{\mathcal{I}}, \ldots, x_{K}^{\mathcal{I}}\right]^{T} \\
{[\mathbf{J}]_{p, q} } & \doteq\left\{\begin{array}{ll}
1, & p-q=\frac{N_{S s}}{2} \\
0, & p-q \neq \frac{N_{s s}}{2}
\end{array} \text { with }|p, q| \leq M\right.
\end{aligned}
$$

where matrix $\mathbf{J}$ is a shift-matrix for modeling the $N_{s s} / 2$ time offset in the quadrature component. Note that $N_{s s} / 2$ is required to be an integer value in (13). Next, the following assumptions are considered.

AS1b) The transmitted symbols $\left\{x_{n}^{\mathcal{R}}, x_{n}^{\mathcal{I}}\right\}$ are real-valued i.i.d. zero mean with variance $\mathrm{E}\left[\left(x_{n}^{\mathcal{R}}\right)^{2}\right]=$ $\mathrm{E}\left[\left(x_{n}^{\mathcal{I}}\right)^{2}\right]=1 / 2$.

AS2b) The transmission pulse shape filter $g(k)$ can be given by a square root raised cosine (SQRRC) pulse, a rectangular (RECT) pulse, or a sinusoisal (SIN) pulse for encompassing different types of staggered modulations. ${ }^{2}$

AS3b) The carrier-phase offset $\theta_{0}$ is assumed to be an unknown deterministic parameter.

AS4b) The normalized carrier-frequency error $\nu$ is assumed to be sufficiently small so as to be negligible, i.e., $\nu=0 .{ }^{3}$

AS5b) The observation interval is asymptotically large, that is, $(K \rightarrow \infty)$ and $(M \rightarrow \infty)$.

\section{The Complex and Noncomplex Cyclic Autocorrelation \\ Functions}

Cyclostationary statistics (CSS) are the basis for a wide range of timing estimators proposed in the literature [2], most of them derived following some ad hoc or heuristic reasonings. For the case under study, the CSS of the received signal allows us to

\footnotetext{
${ }^{2}$ Note that the MSK modulation can also be viewed as a staggered modulation with a sinusoidal pulse shape [12].

${ }^{3} \mathrm{AS} 3 \mathrm{~b}$ ) and AS4b) are the main differences between the analysis for nonoffset and offset modulations. (See AS3a) and AS4a) in Section II-A.)
}

express the ML timing estimation problem in a more valuable and systematic framework. In this sense, the cyclostationarybased formulation [13] is adopted in this paper by means of the use of the cyclic autocorrelation function (CAF).

The most common definition of the CAF is the so-called complex-conjugate CAF (CC-CAF) defined as follows:

$$
R_{x *}^{\alpha}(m)=\lim _{M \rightarrow \infty} \frac{1}{2 M+1} \sum_{k=-M}^{M} x^{*}(k) x(k+m) e^{-j 2 \pi \alpha k}
$$

where the CC-CAF is evaluated at the cycle-frequency $\alpha$ and time-lag $m$, and $(\cdot)^{*}$ is the complex conjugate operator. However, as it is already pointed out in [14], the noncomplex conjugate CAF (NCC-CAF) defined by

$$
R_{x}^{\alpha}(m)=\lim _{M \rightarrow \infty} \frac{1}{2 M+1} \sum_{k=-M}^{M} x(k) x(k+m) e^{-j 2 \pi \alpha k}
$$

can also be useful in some kind of complex signal analysis. In particular, we will show that the NCC-CAF plays a major role in the problem of joint phase and timing estimation for offset modulations. For this type of modulation format, the noncomplex second-order CSS in (15) are able to retain the phase information irrespective of the symbol constellation structure, which is a valuable result for the NDA parameter estimation problem. For this reason, both the CC-CAF and the NCC-CAF are employed along the paper. For finite length observation intervals, the exact CC-CAF in (14) and the NCC-CAF in (15) can be replaced respectively, by the unbiased estimators given by

$$
\begin{aligned}
\widehat{R}_{x *}^{\alpha}(m) & =\frac{1}{2 M+1} \sum_{k=-M}^{M} x^{*}(k) x(k+m) e^{-j 2 \pi \alpha k} \\
\widehat{R}_{x}^{\alpha}(m) & =\frac{1}{2 M+1} \sum_{k=-M}^{M} x(k) x(k+m) e^{-j 2 \pi \alpha k}
\end{aligned}
$$

which are consistent and efficient estimators of the exact CC-CAF and NCC-CAF for any $\alpha$ and $m$ [15].

\section{UML TIMING ESTIMATION}

The Stochastic or UML criterion provides an almost common framework to describe a wide range of synchronization techniques [1]. The NDA parameter estimation implies the evaluation of the marginal statistics with respect to the nuisance parameters included in vector $\Theta$. For the case under study, the NDA ML symbol-timing error estimation is obtained as

$$
\widehat{\tau}=\arg \max _{\tau} \mathrm{E}_{\nu} \mathrm{E}_{\theta_{0}} \mathrm{E}_{\mathbf{x}}[\Lambda(\mathbf{r} \mid \Theta ; \mathbf{x})]
$$

where $\Lambda(\mathbf{r} \mid \Theta ; \mathbf{x})$ is the Likelihood function of the problem.

We will first focus on the case of linear modulations with nonoffset format as described in Section II-A. The likelihood function for the AWGN channel is then given by

$$
\Lambda(\mathbf{r} \mid \Theta ; \mathbf{x})=C_{1} \exp \left(-\frac{1}{\sigma_{w}^{2}}\|\mathbf{r}-\mathbf{A}(\boldsymbol{\Theta}) \mathbf{x}\|^{2}\right)
$$

where $C_{1}$ is an irrelevant constant. As mentioned before, the main drawback of the UML approach is that the computation of 
the marginal statistics given in (18) poses insurmountable obstacles for the derivation of a closed-form expression. To circumvent this important limitation, a low-SNR analysis is often referred in the literature [16], [17]. In this case, it is shown that the expectation with respect to the unknown nuisance parameters can be easily performed. Under the low-SNR assumption, the Taylor expansion of (19) up to the quadratic term is given by $[1$, eq. (1.3.6)]

$$
\Lambda(\mathbf{r} \mid \boldsymbol{\Theta} ; \mathbf{x}) \approx C_{1}\left[1+\frac{2}{\sigma_{w}^{2}} \chi(\mathbf{r} ; \boldsymbol{\Theta} ; \mathbf{x})+\frac{2}{\sigma_{w}^{4}} \chi^{2}(\mathbf{r} ; \boldsymbol{\Theta} ; \mathbf{x})\right]
$$

with

$$
\begin{aligned}
\chi(\mathbf{r} ; \boldsymbol{\Theta} ; \mathbf{x}) & \doteq \operatorname{Re}\left[\mathbf{x}^{H} \mathbf{A}^{H} \mathbf{r}\right], \\
\chi^{2}(\mathbf{r} ; \boldsymbol{\Theta} ; \mathbf{x}) & =\frac{1}{2} \operatorname{Re}\left[\mathbf{r}^{T} \mathbf{A}^{*} \mathbf{x}^{*} \mathbf{x}^{H} \mathbf{A}^{H} \mathbf{r}\right]+\frac{1}{2} \mathbf{r}^{H} \mathbf{A} \mathbf{x} \mathbf{x}^{H} \mathbf{A}^{H} \mathbf{r} .
\end{aligned}
$$

The expectation with respect to the nuisance parameters in (21)-(22) is then given by

$$
\begin{aligned}
\mathrm{E}_{\mathbf{x}}[\chi(\mathbf{r} ; \boldsymbol{\Theta} ; \mathbf{x})] & =0 \\
\mathrm{E}_{\nu} \mathrm{E}_{\theta_{0}} \mathrm{E}_{\mathbf{x}}\left[\chi^{2}(\mathbf{r} ; \boldsymbol{\Theta} ; \mathbf{x})\right] & =\frac{1}{2} \mathbf{r}^{H} \mathrm{E}_{\nu}\left[\mathbf{A} \mathbf{A}^{H}\right] \mathbf{r}
\end{aligned}
$$

where we have taken into consideration the assumption in AS1a).

Given a prior distribution for the carrier-frequency error $\nu$, it is shown in [18] that the $(p, q)$ entries of the outer-product $\mathbf{A A}^{H}$ are asymptotically given by (25) at the bottom of this page. These entries can be efficiently expressed in matrix notation as

$$
\lim _{K \rightarrow \infty} \mathrm{E}_{\nu}\left[\mathbf{A A}^{H}\right]=\left[\mathbf{G}_{\tau} \mathbf{G}_{\tau}^{H}\right] \odot \mathbf{V}
$$

with $\odot$ the Schur-Hadamard (i.e., component-wise) product, and

$$
\begin{aligned}
{\left[\mathbf{G}_{\tau} \mathbf{G}_{\tau}^{H}\right]_{p, q} } & =\sum_{n=-\infty}^{\infty} g\left(p-n N_{s s}-\tau\right) g^{*}\left(q-n N_{s s}-\tau\right) \\
{[\mathbf{V}]_{p, q} } & =\int_{-N_{s s} / 2}^{N_{s s} / 2} e^{j\left(2 \pi / N_{s s}\right) \nu(p-q)} f_{\nu}(\nu) d \nu \\
& =\int_{-\pi}^{\pi} e^{j \bar{\omega}_{\epsilon}(p-q)} f_{\bar{\omega}_{\epsilon}}\left(\bar{\omega}_{\epsilon}\right) d \bar{\omega}_{\epsilon}=\Phi_{\bar{\omega}_{\epsilon}}(p-q)
\end{aligned}
$$

for $|p, q| \leq M$. According to [19, p. 115], we find that the expression for the $(p, q)$ entries of the Doppler spreading matrix $\mathbf{V}$ is given by the characteristic function associated to the carrier-frequency error pdf, that is

$$
\Phi_{\bar{\omega}_{\epsilon}}(\lambda)=\int_{-\infty}^{\infty} f_{\bar{\omega}_{\epsilon}}\left(\bar{\omega}_{\epsilon}\right) e^{j \lambda \bar{\omega}_{\epsilon}} d \bar{\omega}_{\epsilon}
$$

where the substitution $\bar{\omega}_{\epsilon}=\left(2 \pi / N_{s s}\right) \nu$ has been applied to obtain an expression which purely depends on the distance between the matrix elements in $\mathbf{V}$.

By substituting (23), (24), and (26) into (20), we find that the UML cost function can be expressed up to an irrelevant constant factor by

$$
\Lambda(\mathbf{r} \mid \tau) \propto \operatorname{Tr}\left(\left[\left(\mathbf{G}_{\tau} \mathbf{G}_{\tau}^{H}\right) \odot \mathbf{V}\right] \widehat{\mathbf{R}}\right)
$$

where $\widehat{\mathbf{R}} \doteq \mathbf{r r}^{H}$ stands for the sample covariance matrix of the received signal, $\operatorname{Tr}(\cdot)$ for the trace operator and $\propto$ for the proportional to operator.

Nevertheless, the Likelihood cost function in (30) can be further simplified by exploiting the CSS structure of the received signal. As it is shown in Appendix I, for bandlimited pulse shapes AS2a) and for infinite observation intervals AS5a), the entries along the $m$-th diagonal of matrix $\mathbf{G}_{\tau} \mathbf{G}_{\tau}^{H}$ are given by

$$
\left[\mathbf{G}_{\tau} \mathbf{G}_{\tau}^{H}\right]_{k+m, k}=\frac{2 M+1}{N_{s s}} \sum_{n=-1}^{+1} R_{g *}^{n / N_{s s}}(m) e^{j\left(2 \pi / N_{s s}\right)\left(k-\tau_{c} / T_{s}\right) n}
$$

It should be noted that the zero cycle-frequency does not provide any information about the parameter of interest $\tau_{c}$, so this cycle-frequency can be ignored. By assuming a large enough observation interval, it is shown in Appendix II that the trace in (30) can be expanded as follows:

$$
\begin{aligned}
\Lambda(\mathbf{r} \mid \tau) & \propto \sum_{p=-M}^{M} \sum_{q=-M}^{M}\left[\left(\mathbf{G}_{\boldsymbol{\tau}} \mathbf{G}_{\boldsymbol{\tau}}{ }^{H}\right) \odot \mathbf{V}\right]_{p, q}[\widehat{\mathbf{R}}]_{q, p} \\
& \propto \operatorname{Re}\left[e^{j(2 \pi / T) \tau_{c}} \sum_{m=-M}^{M} \Phi_{\bar{\omega}_{\epsilon}}(m)\left[R_{g *}^{1 / N_{s s}}(m)\right]^{*} R_{r *}^{1 / N_{s s}}(m)\right]
\end{aligned}
$$

where $R_{r *}^{1 / N_{s s}}(m)$ is the CC-CAF of the received samples.

Although the expression in (33) is the general formulation of the problem for any potential distribution of the carrier-frequency error, there are two particular cases for which we will concentrate our attention further on in Section IV. The analysis for the maximum carrier-frequency uncertainty will be performed in Section IV-A whereas the case of no carrier-frequency uncertainty will be addressed in Section IV-B. The implications of these hypothesis and their relationship with the OM timing estimation problem will be presented at the end of Section IV.

$$
\begin{aligned}
\lim _{K \rightarrow \infty} \mathrm{E}_{\nu}\left[\left[\mathbf{A A}^{H}\right]_{p, q}\right] & =\int_{-N_{s s} / 2}^{N_{s s} / 2}\left(\sum_{n=-\infty}^{\infty} g\left(p-n N_{s s}-\tau\right) g^{*}\left(q-n N_{s s}-\tau\right) e^{j\left(2 \pi / N_{s s}\right) \nu(p-q)}\right) f_{\nu}(\nu) d \nu \\
& =[\mathbf{V}]_{p, q}\left[\mathbf{G}_{\tau} \mathbf{G}_{\tau}^{H}\right]_{p, q}
\end{aligned}
$$




\section{RELATIONSHIP BETWEEN UML AND OERDER \& MEYR TIMING ESTIMATION}

The UML cost function derived in (33) provides a general framework to derive blind feedforward timing estimators. Notice that (33) proves that the second-order CSS of the received signal can be exploited for optimal timing estimation in low-SNR scenarios. In fact, from (33) it is possible to obtain the well-known and heuristically derived OM timing estimator [9] but also to generalize this partial result to the case of any carrier-frequency error distribution. Therefore, the link between the ML timing estimation and CSS-based timing estimators such as the OM method is finally established. This result complements the partial ML derivation in [1] and the approach presented in [10], [20] where the OM estimator was shown to asymptotically achieve the best performance among the class of estimators based on second-order statistics.

We will now particularly focus on the following two cases: the case of maximum carrier-frequency error uncertainty and the case of no carrier-frequency error uncertainty.

\section{A. Maximum Carrier Frequency Error Uncertainty}

This case corresponds to those scenarios in which the carrier-frequency error can take any value within the whole Nyquist sampling bandwidth. In this case, the most common approach is to consider a uniform distribution for the random carrier-frequency error $\nu$ in order to reflect our complete lack of knowledge about this nuisance parameter, that is

$$
\begin{aligned}
f_{\nu}(\nu) & =\frac{1}{N_{s s}},|\nu|<\frac{N_{s s}}{2} \\
\Phi_{\bar{\omega}_{\epsilon}}(m) & =\Phi_{\nu}\left(\frac{2 \pi}{N_{s s}} m\right)=\delta(m)
\end{aligned}
$$

where $\delta(m)$ is the Kronecker delta function. ${ }^{4}$ In this way, the Doppler spreading matrix $\mathbf{V}$ in (32) turns out to be the identity matrix, $\mathbf{V}=\mathbf{I}$. By substituting (35) into (33), the asymptotic UML cost function results in

$$
\Lambda(\mathbf{r} \mid \tau) \propto \operatorname{Re}\left[e^{j(2 \pi / T) \tau_{c}}\left[R_{g *}^{1 / N_{s s}}(0)\right]^{*} R_{r *}^{1 / N_{s s}}(0)\right] .
$$

Since the pulse shape $g(k)$ is known and symmetric, $R_{g *}^{1 / N_{s s}}(0)$ is a known and real-valued constant. Thus, the asymptotic symbol-timing error $\tau_{c}$ is given by the phase of the lag-term $R_{r *}^{1 / N_{s s}}(0)$ as follows:

$$
\tau_{c}=-\frac{T}{2 \pi} \arg \left\{R_{r *}^{1 / N_{s s}}(0)\right\} .
$$

However, when a finite set of $L_{0}=2 M_{0}+1$ received samples is available, an estimate $\widehat{\tau}_{c}$ of this timing error can be obtained by performing the sample estimate of the CC-CAF, namely, $\widehat{R}_{r *}^{1 / N_{s s}}(0)$, as indicated in (16). The resulting timing estimator is finally given by

$$
\widehat{\tau}_{c}=-\frac{T}{2 \pi} \arg \left\{\frac{1}{2 M_{0}+1} \sum_{k=-M_{0}}^{M_{0}}|r(k)|^{2} e^{-j\left(2 \pi / N_{s s}\right) k}\right\} .
$$

\footnotetext{
${ }^{4}$ The symbol $\delta(x)$ denotes both the Dirac delta function and the Kronecker delta depending on whether $x$ is a continuous-time or a discrete-time variable, respectively.
}

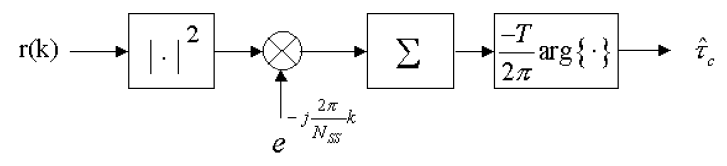

Fig. 1. Block diagram for the asymptotic low-SNR UML symbol-timing estimator for maximum carrier-frequency uncertainty.

The structure of the estimator in (38) is depicted in Fig. 1 for clarity. It can be seen that the information regarding the symboltiming error is contained in the complex envelope of the incoming signal, which exhibits a periodic behavior due to the cyclostationary property of linear modulations.

Hence, the UML timing estimator in (38) is based on performing the discrete Fourier transform (DFT) at the frequency bin corresponding to the symbol rate, whose phase content is providing the timing error information. Due to the frequency error uncertainty, the solution found in (38) suggests that the received signal has to be directly processed without any pre-filtering stage, exploiting only the cyclostationary structure of the instantaneous complex envelope of the incoming signal.

\section{B. No Carrier Frequency Error Uncertainty}

This case corresponds to those scenarios in which there is a perfect knowledge about the carrier-frequency error. Thus, it is assumed that either the carrier-frequency error has been compensated in a previous stage or that it is sufficiently small so as to be negligible compared to the signaling rate.

In this case, the corresponding pdf and the associated characteristic function for the carrier-frequency error are given by

$$
\begin{aligned}
f_{\nu}(\nu) & =\delta(\nu) \\
\Phi_{\bar{\omega}_{\epsilon}}(m) & =\Phi_{\nu}\left(\frac{2 \pi}{N_{s s}} m\right)=1, \text { for all } m
\end{aligned}
$$

with $\delta(\nu)$ the Dirac delta function. For this case, the Doppler spreading matrix $\mathbf{V}$ in (28) and (32) turns out to be an all-ones matrix, that is, $[\mathbf{V}]_{p, q}=1$ for any $(p, q)$ entry. By substituting (40) into (33) we find that the ML function becomes

$$
\Lambda(\mathbf{r} \mid \tau) \propto \operatorname{Re}\left[e^{j(2 \pi / T) \tau_{c}} \sum_{m=-M}^{M}\left[R_{g *}^{1 / N_{s s}}(m)\right]^{*} R_{r *}^{1 / N_{s s}}(m)\right] .
$$

In (41), important insights can be gained by performing the analysis into the frequency domain, allowing us to relate once again the UML formulation with the OM timing recovery. For this purpose, let us recall the Parseval-Plancherel's theorem [21]

$$
\sum_{k=-\infty}^{\infty} x(k) y^{*}(k)=\frac{1}{2 \pi} \int_{-\pi}^{\pi} X(\omega) Y^{*}(\omega) d \omega
$$

where $X(\omega)$ and $Y(\omega)$ are the Fourier transforms of the discrete-time signals $x(k)$ and $y(k)$, respectively. That is, for the case of $x(k)$ we have that

$$
X(\omega)=\sum_{k=-\infty}^{\infty} x(k) e^{-j \omega k}
$$


As in [22], the CC-CAF of the sequence $x(k)$ can be asymptotically expressed as

$$
R_{x *}^{\alpha}(m)=\frac{1}{2 \pi} \int_{-\pi}^{\pi} X(\omega) X^{*}(\omega-2 \pi \alpha) e^{j \omega m} d \omega
$$

It is worth noting that an oversampling factor on the signals $x(k)$ and $y(k)$ is required for avoiding the aliasing effects in (44). In particular, for $\alpha=1 / N_{s s}$ and recalling the bandlimited nature of the signals under consideration, it is found that a value $N_{s s} \geq$ 3 is required. This guarantees that $X(\omega-2 \pi \alpha)$ is equivalent to $X_{c}(\omega-2 \pi \alpha)$, being the latter the Fourier transform of the continuous-time signal $x(t) e^{j 2 \pi \alpha t}$ (see [23] and [24]). Otherwise, for $N_{s s}=2$, the discrete-time spectrum $X(\omega-2 \pi \alpha)$ is aliased. Substituting (44) into (41) for the case of $R_{g *}^{1 / N_{s s}}(m)$ and $R_{r *}^{1 / N_{s s}}(m)$, we can find that

$$
\begin{gathered}
\Lambda(\mathbf{r} \mid \tau) \\
\propto \operatorname{Re}\left[e^{j(2 \pi / T) \tau_{c}} \int_{-\pi}^{\pi} \int_{-\pi}^{\pi} G^{*}\left(\omega_{1}\right) R\left(\omega_{2}\right) G\left(\omega_{1}-\frac{2 \pi}{N_{s s}}\right)\right. \\
\left.R^{*}\left(\omega_{2}-\frac{2 \pi}{N_{s s}}\right) \Psi\left(\omega_{1}, \omega_{2}\right) d \omega_{1} d \omega_{2}\right]
\end{gathered}
$$

with

$$
\begin{aligned}
\Psi\left(\omega_{1}, \omega_{2}\right) & =\lim _{M \rightarrow \infty} \sum_{m=-M+1}^{M-1} e^{j\left(\omega_{2}-\omega_{1}\right) m} \\
& =\lim _{M \rightarrow \infty} \frac{\sin \left(\left(\omega_{2}-\omega_{1}\right) \frac{2 M-1}{2}\right)}{\sin \left(\left(\omega_{2}-\omega_{1}\right) \frac{1}{2}\right)} \\
& =2 \pi \sum_{n=-\infty}^{\infty} \delta\left(\omega_{2}-\omega_{1}-n 2 \pi\right)
\end{aligned}
$$

where $\Psi\left(\omega_{1}, \omega_{2}\right)$ is a periodic function on $\left(\omega_{2}-\omega_{1}\right)$ and $\delta(\cdot)$ is the Dirac delta function. However, as the signals under consideration are bandlimited, it is necessary to set $\omega_{2}=\omega_{1}$ and $n=0$ in (48) for (45) to be different from zero. Hence, the UML cost function simplifies to

$$
\begin{aligned}
\Lambda(\mathbf{r} \mid \tau) & \propto \operatorname{Re}\left[e^{j(2 \pi / T) \tau_{c}} \int_{-\pi}^{\pi} Y(\omega) Y^{*}\left(\omega-\frac{2 \pi}{N_{s s}}\right) d \omega\right] \\
& =\operatorname{Re}\left[e^{j(2 \pi / T) \tau_{c}} R_{y *}^{1 / N_{s s}}(0)\right]
\end{aligned}
$$

where $Y(\omega)$ is the Fourier transform of the received samples at the matched filter output, that is

$$
\begin{aligned}
Y(\omega) & =R(\omega) G^{*}(\omega) \\
y(k) & =r(k) * g^{*}(-k)
\end{aligned}
$$

where $*$ stands for the linear convolution operator. Finally, it is not difficult to show from (50) that the symbol-timing error $\tau_{c}$ is obtained from the phase information of the lag $R_{y *}^{1 / N_{s s}}(0)$, or in other words

$$
\tau_{c}=-\frac{T}{2 \pi} \arg \left\{R_{y *}^{1 / N_{s s}}(0)\right\} .
$$

When a finite set of $L_{0}=2 M_{0}+1$ received samples is available, an estimate $\widehat{\tau}_{c}$ of this timing error can be obtained from

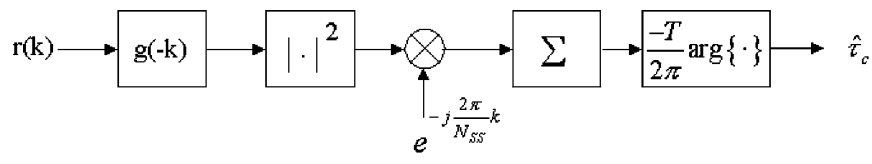

Fig. 2. Block diagram for the asymptotic low-SNR UML symbol-timing estimator for minimum carrier-frequency uncertainty.

the sample estimate of the CC-CAF lag $\widehat{R}_{y *}^{1 / N_{s s}}(0)$; finally, the resulting UML timing estimator is given by

$$
\widehat{\tau}_{c}=-\frac{T}{2 \pi} \arg \left\{\frac{1}{2 M_{0}+1} \sum_{k=-M_{0}}^{M_{0}}|r(k) * g(-k)|^{2} e^{-j\left(2 \pi / N_{s s}\right) k}\right\}
$$

and depicted in Fig. 2.

After the analysis, we can conclude the following.

1) When no frequency error is present at the received signal, the optimal low-SNR timing estimate only considers the impact of the thermal noise, ignoring the influence of the self-noise (i.e., the data-dependent jitter). This fact can be inferred from (54), where the optimal solution requires the filtering of the received data by the matched filter of the transmitted pulse shape. For moderate to high-SNR values, the most important degradation is not due to the thermal noise but to the self-noise. In this sense, it can be shown that when the SNR is moderate or high, the self-noise can be reduced by the adoption of other types of prefiltering stages becoming the matched filtering a suboptimal approach [5]-[8].

2) The timing estimation illustrated in (54) can be performed with an oversampling factor $N_{s s} \geq 3$. This is consistent with the results derived in [10] and [24] based on an expression similar to (44), but contrary to the traditional belief that the OM algorithm requires at least an oversampling factor of $N_{s s}=4$ in the received data (see [17, Section 7.6.2] and [9, Section II]).

\section{Generalization of the Oerder And Meyr (OM) ALgORITHM TO JOINT PHASE AND TIMING ESTIMATION FOR STAGGERED MODULATIONS}

The UML approach already introduced in Section III can also be applied to the case of the offset modulations introduced in Section II-B. In this case, the exploitation of the CSS allows the joint estimation of the carrier-phase and the symbol-timing errors. This is a basic difference between the analysis of the problem for nonoffset and offset modulations.

As for Section III, the likelihood function for staggered modulations becomes

$$
\Lambda(\mathbf{r} \mid \boldsymbol{\Theta} ; \mathbf{x})=C_{1} \exp \left(-\frac{1}{\sigma_{w}^{2}}\left\|\mathbf{r}-\mathbf{A}(\boldsymbol{\Theta}) \mathbf{x}_{\mathcal{R}}-j \mathbf{J} \mathbf{A}(\boldsymbol{\Theta}) \mathbf{x}_{\mathcal{I}}\right\|^{2}\right)
$$

where $\mathbf{x}=\left\{\mathbf{x}_{\mathcal{R}}, \mathbf{x}_{\mathcal{I}}\right\}$ are the in-phase and the staggered quadrature symbols and $C_{1}$ is an irrelevant constant. By applying the low SNR assumption, the Likelihood function can be approximated by

$$
\Lambda(\mathbf{r} \mid \boldsymbol{\Theta} ; \mathbf{x}) \approx C\left[1+\frac{2}{\sigma_{w}^{2}} \chi(\mathbf{r} ; \boldsymbol{\Theta} ; \mathbf{x})+\frac{2}{\sigma_{w}^{2}} \chi^{2}(\mathbf{r} ; \boldsymbol{\Theta} ; \mathbf{x})\right]
$$


with

$$
\begin{aligned}
\chi(\mathbf{r} ; \boldsymbol{\Theta} ; \mathbf{x})= & \operatorname{Re}\left[\mathbf{x}_{\mathcal{R}}{ }^{T} \mathbf{A}^{H} \mathbf{r}\right]+\operatorname{Im}\left[\mathbf{x}_{\mathcal{I}^{T}} \mathbf{A}^{H} \mathbf{J}^{T} \mathbf{r}\right] \\
\chi^{2}(\mathbf{r} ; \boldsymbol{\Theta} ; \mathbf{x})= & \frac{1}{4} \operatorname{Re}\left[\mathbf{r}^{T} \mathbf{A}^{*} \mathbf{A}^{H} \mathbf{r}-\mathbf{r}^{T} \mathbf{J} \mathbf{A}^{*} \mathbf{A}^{H} \mathbf{J}^{T} \mathbf{r}\right] \\
& +\frac{1}{4}\left(\mathbf{r}^{H} \mathbf{A} \mathbf{A}^{H} \mathbf{r}+\mathbf{r}^{H} \mathbf{J} \mathbf{A} \mathbf{A}^{H} \mathbf{J}^{T} \mathbf{r}\right)
\end{aligned}
$$

In the sequel, we will denote by $\mathrm{E}_{\mathbf{x}}[\cdot]$ the joint expectation operator with respect to the in-phase and the quadrature symbols, (i.e., $\mathrm{E}_{\mathbf{x}} \doteq \mathrm{E}_{\mathbf{x}_{\mathcal{R}}} \mathrm{E}_{\mathbf{x}_{\mathcal{T}}}$ ). According to AS1b), we have from (57) that $\mathrm{E}_{\mathbf{x}}[\chi(\mathbf{r} ; \boldsymbol{\Theta} ; \mathbf{x})]=0$, and then the likelihood function simplifies to

$$
\Lambda\left(\mathbf{r} \mid \theta_{0}, \tau\right) \propto \mathrm{E}_{\mathbf{x}}\left[\chi^{2}(\mathbf{r} ; \boldsymbol{\Theta} ; \mathbf{x})\right]
$$

Moreover, from assumptions AS3b) and AS4b), we have that (59) is given by

$$
\begin{aligned}
\mathrm{E}_{\mathbf{x}}\left[\chi^{2}(\mathbf{r} ; \boldsymbol{\Theta} ; \mathbf{x})\right]= & \frac{1}{4} \operatorname{Re}\left[e^{-j 2 \theta_{0}}\left(\mathbf{r}^{T} \mathbf{G}_{\tau}^{*} \mathbf{G}_{\tau}^{H} \mathbf{r}-\mathbf{r}^{T} \mathbf{J} \mathbf{G}_{\tau}^{*} \mathbf{G}_{\tau}^{H} \mathbf{J}^{T} \mathbf{r}\right)\right] \\
& +\frac{1}{4}\left(\mathbf{r}^{H} \mathbf{G}_{\tau} \mathbf{G}_{\tau}^{H} \mathbf{r}+\mathbf{r}^{H} \mathbf{J} \mathbf{G}_{\tau} \mathbf{G}_{\tau}^{H} \mathbf{J}^{T} \mathbf{r}\right) .
\end{aligned}
$$

At this point, it will be useful to define the signals $y_{1}(k)$ and $y_{2}(k)$ as the following matched filter outputs for the received signal $r(k)$ :

$$
\begin{aligned}
& y_{1}(k)=r(k) * g^{*}(-k) \\
& y_{2}(k)=r(k) * g^{*}\left(-k+\frac{N_{s s}}{2}\right) .
\end{aligned}
$$

It is worth noting that both sequences are the same up to a constant time delay between them.

The analysis will be done once again for a long enough observation interval and the asymptotic results derived in Section IV-B will be reused. Following the derivations indicated in Appendix III, we can conclude that

$$
\begin{aligned}
& \mathbf{r}^{H} \mathbf{G}_{\tau} \mathbf{G}_{\tau}^{H} \mathbf{r} \\
& =C_{2} \operatorname{Re}\left[e^{j(2 \pi / T) \tau_{c}} R_{y_{1} *}^{1 / N_{s s}}(0)\right] \\
& \mathbf{r}^{H} \mathbf{J G}_{\tau} \mathbf{G}_{\tau}^{H} \mathbf{J}^{T} \mathbf{r} \\
& =C_{2} \operatorname{Re}\left[e^{j(2 \pi / T) \tau_{c}} R_{y_{2} *}^{1 / N_{s s}}(0)\right] \\
& \mathbf{r}^{T} \mathbf{G}_{\tau}^{*} \mathbf{G}_{\tau}^{H} \mathbf{r} \\
& =C_{3}\left[e^{j(2 \pi / T) \tau_{c}} R_{y_{1}}^{1 / N_{s s}}(0)+e^{-j(2 \pi / T) \tau_{c}} R_{y_{1}}^{-1 / N_{s s}}(0)\right] \\
& \mathbf{r}^{T} \mathbf{J G}_{\tau}^{*} \mathbf{G}_{\tau}^{H} \mathbf{J}^{T} \mathbf{r} \\
& =C_{3}\left[e^{j(2 \pi / T) \tau_{c}} R_{y_{2}}^{1 / N_{s s}}(0)+e^{-j(2 \pi / T) \tau_{c}} R_{y_{2}}^{-1 / N_{s s}}(0)\right]
\end{aligned}
$$

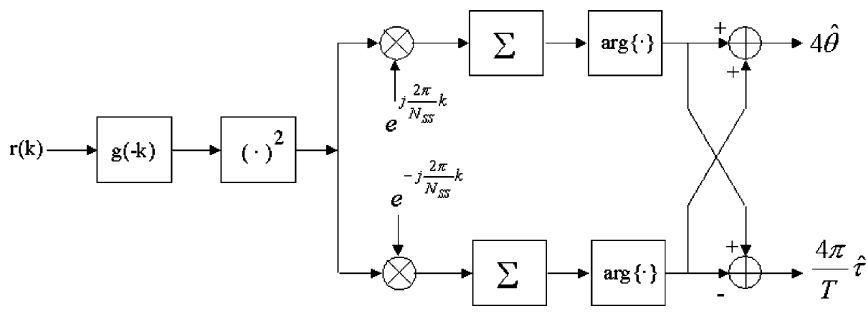

Fig. 3. Block diagram for the asymptotic UML joint phase and timing estimator for offset modulations.

where $C_{2}$ and $C_{3}$ are irrelevant constants. Due to the relative $N_{s s} / 2$ time-offset between the signal $y_{2}(k)$ and the signal $y_{1}(k)$, a phase shift equal to $e^{-j\left(2 \pi / N_{s s}\right)\left(N_{s s} / 2\right)}=-1$ appears in the CC- and NCC-CAF associated to $y_{2}(k)$. Thus

$$
\begin{aligned}
R_{y_{2}}^{-1 / N_{s s}}(0) & =-R_{y_{1}}^{-1 / N_{s s}}(0) \\
R_{y_{2} *}^{1 / N_{s s}}(0) & =-R_{y_{1} *}^{1 / N_{s s}}(0) \\
R_{y_{2}}^{1 / N_{s s}}(0) & =-R_{y_{1}}^{1 / N_{s s}}(0)
\end{aligned}
$$

which implies that the complex conjugate second-order statistics from the received signal do not carry any information related to the synchronization parameters. This is due to the fact that the second term in (60) vanishes. That is, we can replace (68) into (64) resulting in

$$
\mathbf{r}^{H} \mathbf{G}_{\tau} \mathbf{G}_{\tau}^{H} \mathbf{r}+\mathbf{r}^{H} \mathbf{J} \mathbf{G}_{\tau} \mathbf{G}_{\tau}^{H} \mathbf{J}^{T} \mathbf{r}=0
$$

In the same way, by replacing (69) and (67) into (65) and (66), it is possible to see from (60) and (70) that the Likelihood function given in (59) can be finally expressed as shown in (71) at the bottom of the page.

The maximization of the UML cost function in (71) is achieved when the following pair of conditions are jointly satisfied:

$$
\begin{aligned}
& 2 \theta_{0}-\frac{2 \pi}{T} \tau_{c}=\arg \left\{R_{y_{1}}^{1 / N_{s s}}(0)\right\} \\
& 2 \theta_{0}+\frac{2 \pi}{T} \tau_{c}=\arg \left\{R_{y_{1}}^{-1 / N_{s s}}(0)\right\}
\end{aligned}
$$

Solving the above equations with respect $\theta_{0}$ and $\tau_{c}$, we find that the optimal asymptotic low-SNR UML joint phase and timing estimation is then given by

$$
\begin{aligned}
\theta_{0} & =\frac{1}{4}\left[\arg \left\{R_{y_{1}}^{1 / N_{s s}}(0)\right\}+\arg \left\{R_{y_{1}}^{-1 / N_{s s}}(0)\right\}\right] \\
\tau_{c} & =-\frac{T}{4 \pi}\left[\arg \left\{R_{y_{1}}^{1 / N_{s s}}(0)\right\}-\arg \left\{R_{y_{1}}^{-1 / N_{s s}}(0)\right\}\right]
\end{aligned}
$$

an estimation procedure that is depicted in Fig. 3 for clarity. Once again, when a finite set of received samples is available,

$$
\Lambda\left(\mathbf{r} \mid \theta_{0}, \tau\right) \propto \operatorname{Re}\left[e^{-j\left(2 \theta_{0}-(2 \pi / T) \tau_{c}\right)} R_{y_{1}}^{1 / N_{s s}}(0)+e^{-j\left(2 \theta_{0}+(2 \pi / T) \tau_{c}\right)} R_{y_{1}}^{-1 / N_{s s}}(0)\right] .
$$


the exact NCC-CAF can be replaced by its sample estimate $\widehat{R}_{y 1}^{\alpha}$ given by

$$
\widehat{R}_{y 1}^{\alpha}=\frac{1}{2 M_{0}+1} \sum_{k=-M_{0}}^{M_{0}}\left[r(k) * g^{*}(-k)\right]^{2} e^{-j 2 \pi \alpha k} .
$$

Similarly to the case of linear modulations with nonoffset formats, it is worth noting that the ML estimate framework for offset modulations also includes the common OM kernel for the joint phase and timing estimation. This common kernel is composed by a second-order nonlinearity followed by a correlation with a spectral line at the symbol rate. Contrary to linear modulations with nonoffset format, the second-order nonlinearity in offset modulations enables us to provide not only the symbol-timing but also the carrier-phase error. This result is of significant relevance, as the carrier-phase estimation can be performed in a NDA manner by a quadratic estimator irrespective of the symbol constellation. Notice that this property does not hold for linear modulations with nonoffset format, which always require the use of higher order moments for the NDA carrier-phase extraction (e.g., [25]).

\section{SimUlation RESUlts}

This section will try to illustrate the performance achieved by the techniques developed in the present paper. To this end, Section VI-A will be focused on the performance evaluation for the case of nonoffset modulations whereas the case of offset modulations will be considered in Section VI-B.

The reference algorithms in the comparative analysis will be the second order estimators derived in [17, Section 8.4] and [17, Section 8.7] which will be further on denoted as the $M L$-based timing estimators for nonoffset and offset modulations respectively. These estimators were derived following a rigorous ML criterion. In general, this implies a good performance but at the expense of a high computational burden when compared with the proposed estimation techniques in the present paper.

Finally, the solutions given in (54) and (74)-(75) will be generally denoted here as the Square-Law Nonlinearity (SLN) estimators. For short, these estimators will be referred as the nonoffset-SLN and the offset-SLN estimators for the case of nonoffset and offset modulations, respectively.

\section{A. Linear Modulations With Nonoffset Format}

In order to summarize the so-called ML-based timing estimator in [17, Section 8.4], the estimation procedure is given by the following equation:

$$
\widehat{\tau}_{M L}=-\frac{T}{2 \pi} \arg \left\{\sum_{k=0}^{M-1}\left[y_{A A F}(k) e^{-j\left(\pi / N_{s s}\right) k}\right] z(k)\right\}
$$

with $M=N_{s s} L$, and where

$$
\begin{aligned}
z(k) & =\left[y_{A A F}^{*}\left(k_{2}\right) e^{-j\left(\pi / N_{s s}\right) k_{2}}\right] * q(k) \\
q(k) & =\frac{\beta}{\pi} \frac{\cos \left(\frac{\pi \beta k}{N_{s s}}\right)}{1-\left(\frac{2 \beta k}{N_{s s}}\right)^{2}}
\end{aligned}
$$

and $y_{A A F}(k)$ is the output of the anti-aliasing filter.
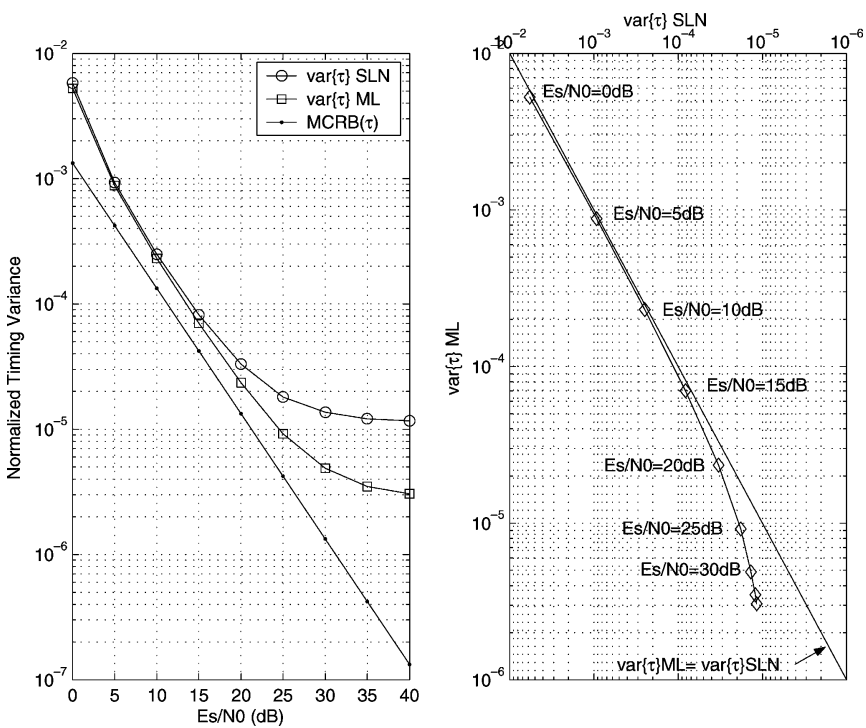

Fig. 4. [16-QAM](Left) Normalized timing variance as a function of $E_{s} / N_{0}$ for $\beta=0.5$. (Right) Comparison between the normalized timing variance for the nonoffset-SLN and the ML-based timing estimator.
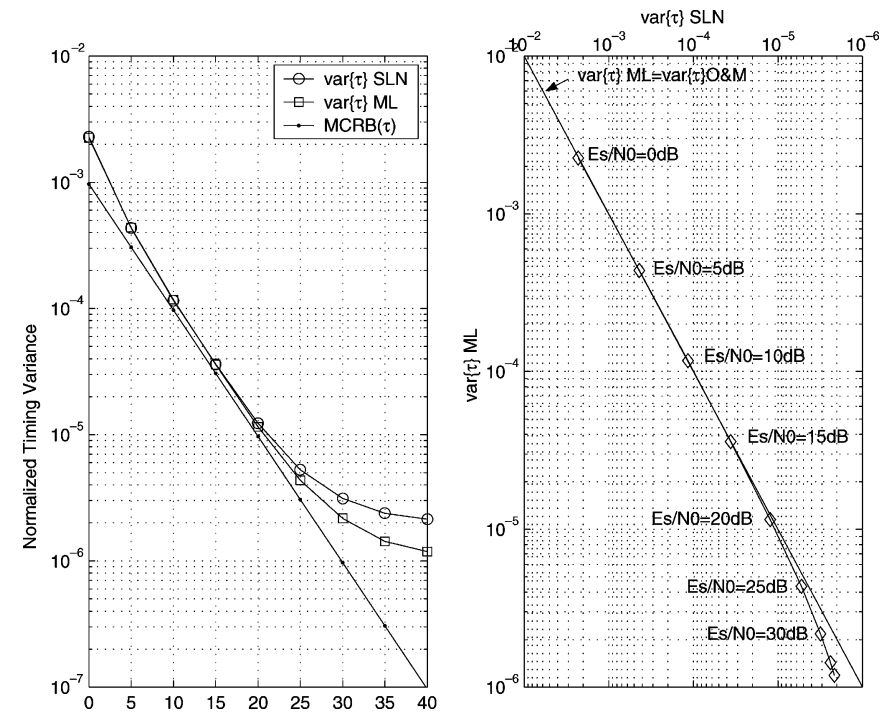

Fig. 5. [16-QAM](Left) Normalized timing variance as a function of $E_{s} / N_{0}$ for $\beta=1.0$. (Right) Comparison between the normalized timing variance for the nonoffset-SLN and the ML-based timing estimator.

Computer simulations for the above ML-based timing estimator in [17, sec.8.4] and for the nonoffset-SLN timing estimator in (54) have been performed for the case of a 16-QAM modulation with $N_{s s}=3$ samples per symbol. The simulation analysis has been carried out as a function of the roll-off factor $\beta$ and for different observation interval lengths $L$ (in number of symbols).

Experiment 1A-Performance Comparison as a Function of the Roll-Off Factor: Figs. 4 and 5 show the normalized timing variance for the nonoffset-SLN and for the ML-based timing estimators with the following two roll-off factors: $\beta=\{0.5,1.0\}$. The symmetric observation interval has been set to $L=101$ symbols corresponding to the one-sided observation interval of $M=50$ symbols. 


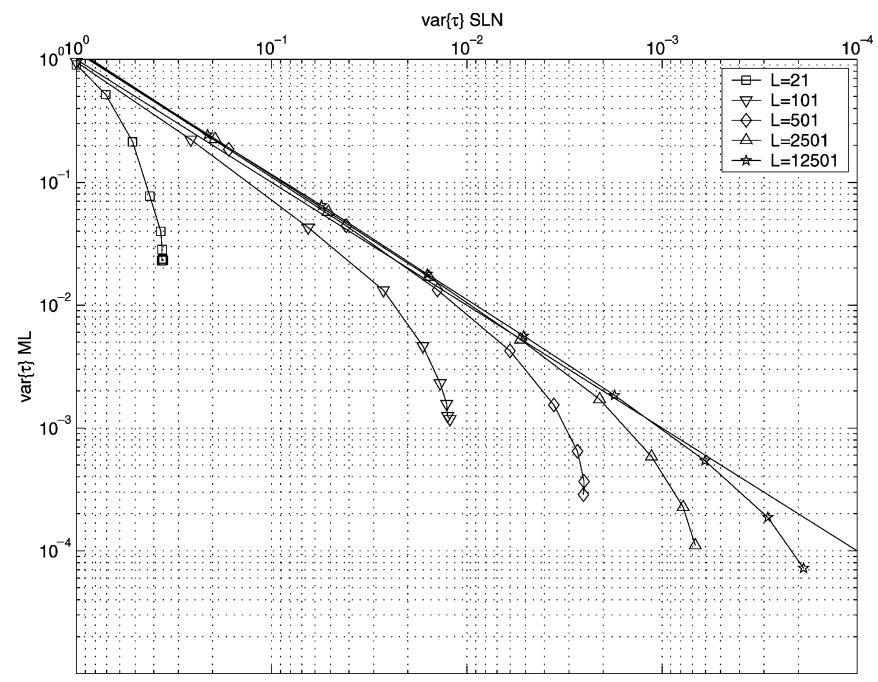

Fig. 6. [16-QAM] Normalized timing variance comparison for the nonoffset-SLN and the ML-based timing estimator as a function of the observation interval $L$, with roll-off factor $\beta=0.1$. For each $L$ plot, both timing variances are computed from $E_{s} / N_{0}=0 \mathrm{~dB}$ to $E_{s} / N_{0}=40 \mathrm{~dB}$ in $5 \mathrm{~dB}$ steps, and normalized to the variance value at $E_{s} / N_{0}=0 \mathrm{~dB}$ for the corresponding $L$.

The left-hand side plots correspond to the timing variance as a function of the $E_{s} / N_{0}$. It can be seen that both timing estimation schemes achieve the same performance for the low $E_{s} / N_{0}$ range as expected by the common low-SNR assumption in the ML derivation. Moreover, this similar behavior of the two techniques is seen to increase as a function of the roll-off factor. This is motivated by the enhancement with the roll-off factor of the amplitude associated to the spectral line at the symbol rate [26, Section 6.5.2]. Finally, in order to compare the behavior of the two techniques, the right hand side plots in Figs. 4 and 5 provide an $\mathrm{X}-\mathrm{Y}$ representation. In this way, it is more evident to notice that although the ML-based scheme always outperforms the nonoffset-SLN technique for the high $E_{s} / N_{0}$ range, their performance coincide in a wide low $E_{s} / N_{0}$ range. For example, for the intermediate case of $\beta=0.5$ in Fig. 4, it is found that the timing variance of both the nonoffset-SLN and the ML-based timing estimator are almost identical up to an $E_{s} / N_{0}=15 \mathrm{~dB}$.

Experiment 2A-Asymptotic Performance: Both timing estimation algorithms are now tested for the range of observation intervals corresponding to $L=$ $\{21,101,501,2501,12501\}$ symbols. The corresponding X-Y plots are now depicted in Fig. 6 for the case of a roll-off factor $\beta=0.1$, which is already a tough case. Each curve in Fig. 6 represents the timing variance from $E_{s} / N_{0}=0 \mathrm{~dB}$ up to $E_{s} / N_{0}=30 \mathrm{~dB}$ (5 dB steps) for all the specified observation intervals $L$. In all the plots, the variance evolution for each curve is always normalized by each associated variance value at $E_{s} / N_{0}=0 \mathrm{~dB}$. In this way, all the curves can be fairly compared in the same axis irrespective of their observation intervals. Therefore, it can be observed in Fig. 6 that both techniques convergence to the same behavior asymptotically with the length of the observation interval.
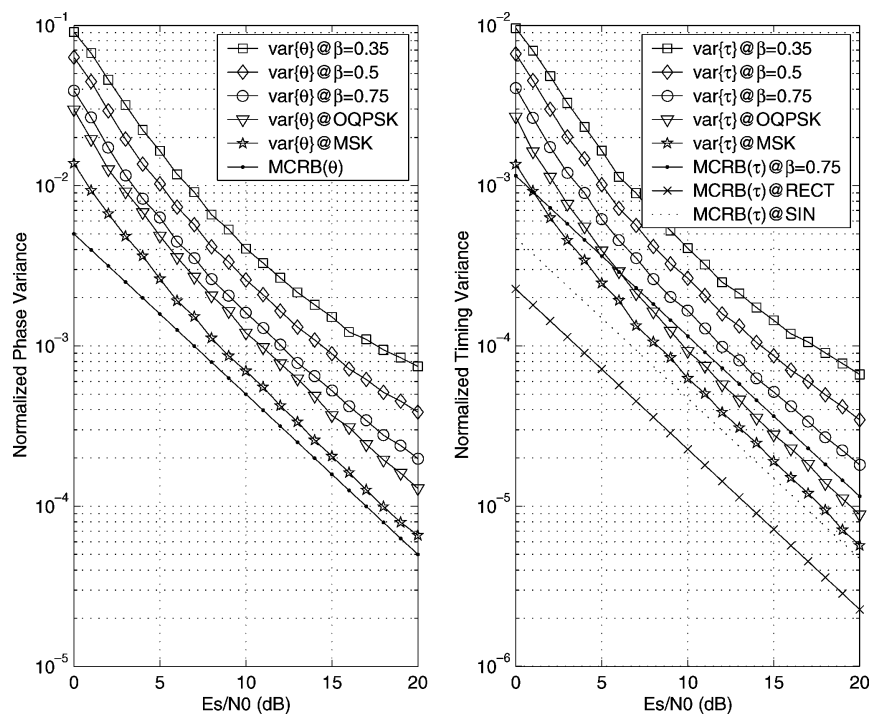

Fig. 7. Phase variance (left) and normalized timing variance (right) for the offset-SLN estimator derived for the case of offset modulation formats.

\section{B. Linear Modulations With Offset Formats}

The offset-SLN estimator given in (74)-(76) will be compared with the ML-based scheme presented in [17, Section 8.7] and [27] which is herein summarized for clarity

$$
\begin{aligned}
& \widehat{\theta}_{M L}=\frac{1}{4}[\arg \{X\}+\arg \{Y\}] \\
& \widehat{\tau}_{M L}=\frac{T}{4 \pi}[-\arg \{X\}+\arg \{Y\}]
\end{aligned}
$$

where

$$
\begin{aligned}
X & =\sum_{k=0}^{M-1}\left[y_{A A F}(k) e^{-j\left(\pi / N_{s s}\right) k}\right] u(k) \\
Y & =\sum_{k=0}^{M-1}\left[y_{A A F}(k) e^{-j\left(\pi / N_{s s}\right) k}\right] v(k) \\
u(k) & =\left[y_{A A F}(k) e^{-j\left(\pi / N_{s s}\right) k}\right] * q(k) \\
v(k) & =\left[y_{A A F}(k) e^{j\left(\pi / N_{s s}\right) k}\right] * q^{*}(k) \\
q(k) & =\frac{\beta}{\pi} \frac{\cos \left(\frac{\pi \beta k}{N_{s s}}\right)}{1-\left(\frac{2 \beta k}{N_{s s}}\right)^{2}} .
\end{aligned}
$$

It is worth noting that the structure of the ML-based estimator in [17, Section 8.7] and [27] follows a similar architecture than the offset-SLN estimator proposed in Section V. However, the latter is once again, a solution with a much lower complexity.

Experiment 1B-Performance of the Offset-SLN Estimator as a Function of the Basic Pulse Shape: The phase and timing variance for the offset-SLN estimator in (74)-(76) is represented in Fig. 7. This figure includes the performance for the case of a 16-OQAM modulation based on the SQRRC pulse, but also for the case of OQPSK modulation based on the RECT pulse and MSK modulation corresponding to the SIN pulse. In Fig. 7, it is interesting to see that when the RECT pulse is adopted, the performance is rather poor compared to the corresponding MCRB. 

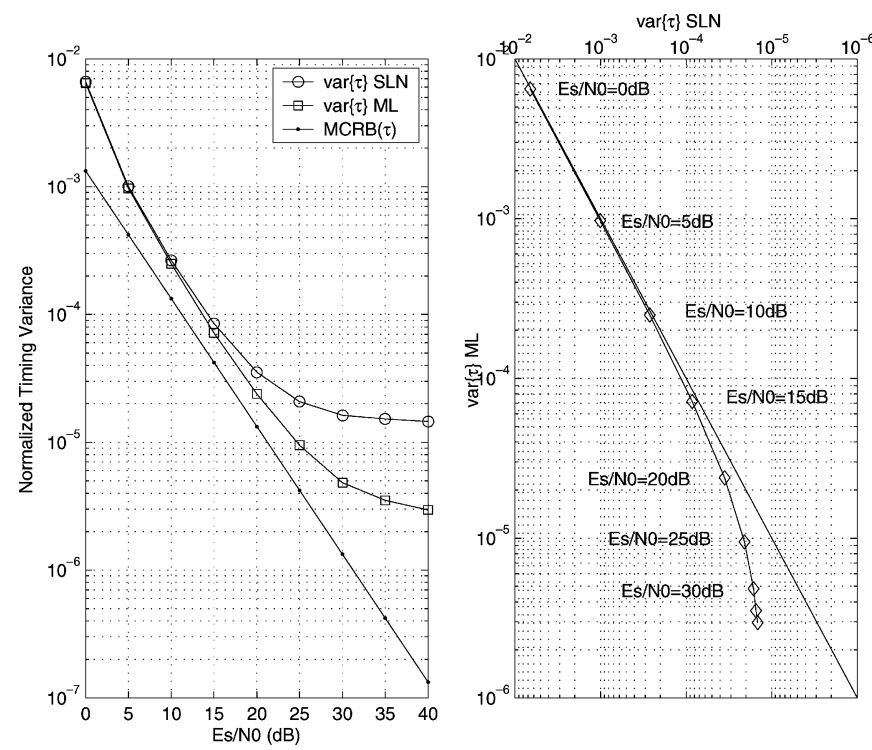

Fig. 8. [16-OQAM] (Left) Normalized timing variance as a function of $E_{s} / N_{0}$ for $\beta=0.5$. (Right) Comparison between the normalized timing variance for the offset-SLN and the ML-based timing estimator derived for offset modulation formats.
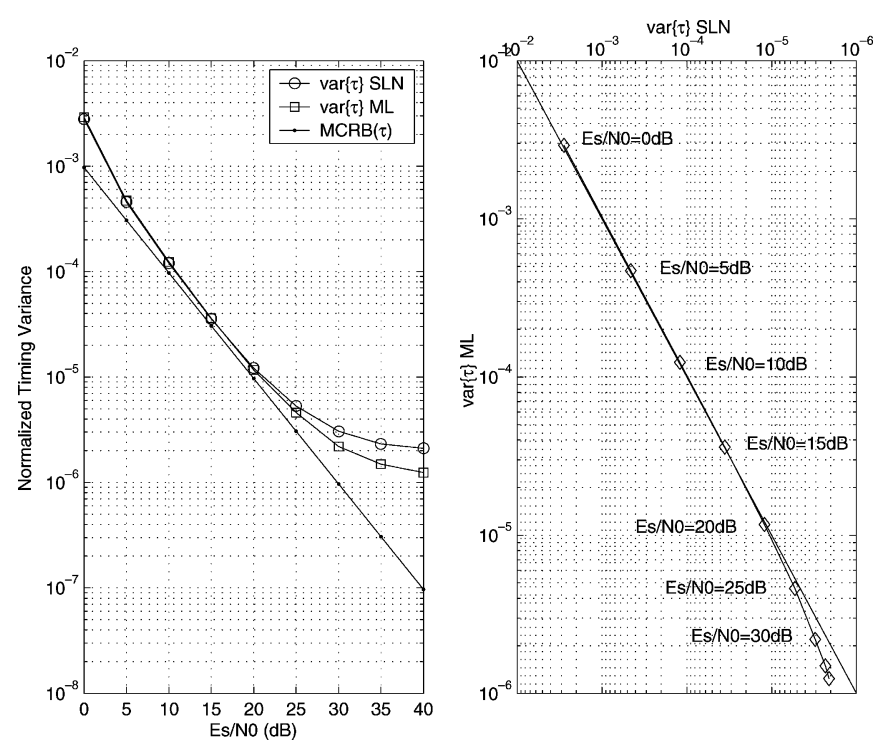

Fig. 9. [16-OQAM] (Left) Normalized timing variance as a function of $E_{s} / N_{0}$ for $\beta=1.0$. (Right) Comparison between the normalized timing variance for the offset-SLN and the ML-based timing estimator derived for offset modulation formats.

This is motivated by the fact that the RECT pulse is not bandlimited and thus, many cycle-frequencies are present in the received data. A similar consideration can be made for the SIN pulse, but because the effective bandwidth is significantly smaller than for the RECT pulse, a smaller degradation is observed.

Experiment 2B-Performance Comparison as a Function of the Roll-Off Factor: The normalized timing variance for both the offset-SLN and the ML-based estimators is depicted in Figs. 8 and 9 for the roll-off factors $\beta=\{0.5,1.0\}$ and a SQRRC pulse. Note that a behavior similar to the case of nonoffset modulations in Figs. 4 and 5 is obtained for different roll-off factors. Moreover, the achieved timing variance seems

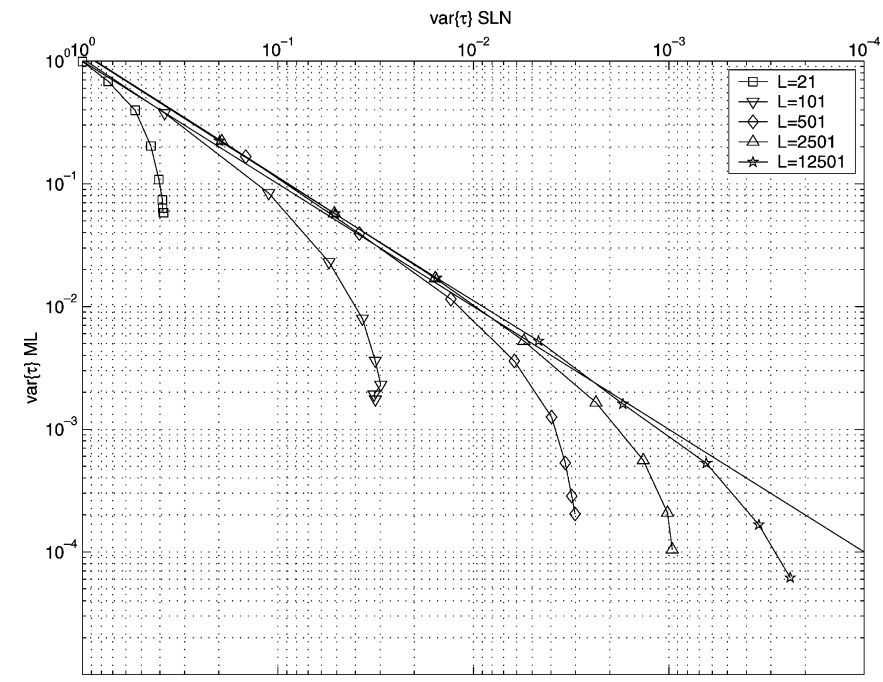

Fig. 10. [16-OQAM] Normalized timing variance comparison for the offset-SLN and the ML-based timing estimator derived for offset modulation formats. The plots are depicted as a function of the observation interval $L$, with a roll-off factor $\beta=0.1$. For each $L$ plot, both timing variances are computed from $E_{s} / N_{0}=0 \mathrm{~dB}$ to $E_{s} / N_{0}=40 \mathrm{~dB}$ in $5 \mathrm{~dB}$ steps, and normalized to the offset-SLN variance value at $E_{s} / N_{0}=0 \mathrm{~dB}$ for the corresponding $L$.

to be almost the same, irrespective of using 16-OQAM or 16-QAM.

Experiment 3B-Asymptotic Performance: The offset-SLN and the ML-based timing estimatiors are now tested for different observation interval lengths. The plot in Fig. 10 also shows a similar behavior to the case of nonoffset modulations previously illustrated in Fig. 6. In particular, it can be seen that the longer the observation interval, the wider the $E_{s} / N_{0}$ range for which both estimators converge.

\section{CONCLUSION}

The problem of symbol-timing estimation has been presented under an analytic and systematic ML approach. The study included the case of linear modulations with nonoffset format, but also the extension to the more general case of linear modulations with offset format (i.e., staggered modulations). In addition, we have assumed the presence of some nuisance parameters such as the carrier-frequency error and the carrier-phase error in the mathematical derivation.

By focusing on the low SNR scenarios, the second-order moments of the received signal are found to provide the sufficient statistics for the problem at hand. In this sense, the cyclostationary property of linear modulations appears to be essential for the synchronization problem, allowing a closed-form expression for the ML estimator. The resulting estimator is found to be robust to the carrier-frequency error uncertainty, but when particularized for the practical cases of interest, a simple scheme is obtained which is based on the well-known and popular OM principle. Moreover, it has been shown that it is possible to extend this approach to the case of offset modulations, where a joint phase and timing estimator is obtained which is also based on the same OM principle.

The relationship between the proposed estimators with more rigorous and complex ML estimators found in the literature is 
addressed. The results confirm that asymptotically, the proposed schemes converge to the rigorous ML estimation. Therefore, the link between ML estimation and the simple OM principle is finally established, providing a new reinterpretation of this well-known algorithm in terms of an optimum synchronization procedure.

\section{APPENDIX I}

EXPLOITING THE CYCLOSTATIONARY PROPERTY OF THE ML COST FunCTION

The outer-product matrix $\mathbf{G}_{\tau} \mathbf{G}_{\tau}^{H}$ plays a major role in the UML approach under analysis in Section III. In particular, noting as $\left[\mathbf{G}_{\tau} \mathbf{G}_{\tau}^{H}\right]_{p, q}$ its $(p, q)$ entries, we see that all the terms along the $m$ th diagonal of matrix $\mathbf{G}_{\tau} \mathbf{G}_{\tau}^{H}$ are given by

$$
\begin{aligned}
{\left[\mathbf{G}_{\tau} \mathbf{G}_{\tau}^{H}\right]_{k+m, k} } & =\sum_{n=-\infty}^{\infty} g\left(k+m-n N_{s s}-\tau\right) g^{*}\left(k-n N_{s s}-\tau\right) \\
& =\widetilde{R}_{g}(k-\tau ; m)
\end{aligned}
$$

where $\widetilde{R}_{g}(k ; m)$ stands for the pulse shape synchronous timevarying autocorrelation, periodically extended with a period of $N_{s s}$ samples

$$
\begin{aligned}
& \widetilde{R}_{g}(k ; m) \doteq \sum_{n=-\infty}^{\infty} R_{g}\left(k-n N_{s s} ; m\right) \\
& R_{g}(k ; m) \doteq g^{*}(k) g(k+m) .
\end{aligned}
$$

It is straightforward to notice the periodic behavior of the diagonal terms in $\mathbf{G}_{\tau} \mathbf{G}_{\tau}^{H}$ due to the fact that

$$
\widetilde{R}_{g}(k-\tau ; m)=\widetilde{R}_{g}\left(k-\tau+l N_{s s} ; m\right)
$$

for any integer $l$. Therefore, these diagonal terms can be expressed by means of a Fourier series expansion as follows:

$$
\left[\mathbf{G}_{\tau} \mathbf{G}_{\tau}^{H}\right]_{k+m, k}=\sum_{n=-1}^{+1} \psi\left(\frac{2 \pi}{N_{s s}} n ; m\right) e^{j\left(2 \pi / N_{s s}\right)\left(k-\tau_{c} / T_{s}\right) n}
$$

where the bandlimited bandwidth of the transmitted pulse shape restricts the number of cycle-frequencies to $n=\{-1,0,+1\}$. Moreover, $\psi\left(\left(2 \pi / N_{s s}\right) n ; m\right)$ are the Fourier coefficients of the pulse shape synchronous time-varying autocorrelation shown in (92)-(93) at the bottom of the page. By applying the change of variable $u \doteq k-n^{\prime} N_{s s}$, and recalling the finite length constraint of (89), we have that

$\psi\left(\frac{2 \pi}{N_{s s}} n ; m\right)=\frac{1}{N_{s s}} \sum_{u=-\infty}^{\infty} g^{*}(u) g(u+m) e^{-j\left(2 \pi / N_{s s}\right) u n}$

so the diagonal terms in $\mathbf{G}_{\tau} \mathbf{G}_{\tau}^{H}$ finally become

$\left[\mathbf{G}_{\tau} \mathbf{G}_{\tau}^{H}\right]_{k+m, k}=\frac{2 M+1}{N_{s s}} \sum_{n=-1}^{+1} R_{g *}^{\left(1 / N_{s s}\right) n}(m) e^{j\left(2 \pi / N_{s s}\right)\left(k-\tau_{c} / T_{s}\right) n}$.

The above considerations for the outer-product matrix $\mathbf{G}_{\tau} \mathbf{G}_{\tau}^{H}$ are also useful for the case of $\mathbf{G}_{\tau}^{*} \mathbf{G}_{\tau}^{H}$. The outer-product matrix $\mathbf{G}_{\tau}^{*} \mathbf{G}_{\tau}^{H}$ is the basis for the joint carrierphase and symbol-timing estimation for offset modulations in Section V, and can be equivalently expressed in a Fourier series as follows,

$\left[\mathbf{G}_{\tau}^{*} \mathbf{G}_{\tau}^{H}\right]_{k+m, k}=\sum_{n=-1}^{+1} \psi_{*}\left(\frac{2 \pi}{N_{s s}} n ; m\right) e^{j\left(2 \pi / N_{s s}\right)\left(k-\tau_{c} / T_{s}\right) n}$

with

$\psi_{*}\left(\frac{2 \pi}{N_{s s}} n ; m\right)=\frac{1}{N_{s s}} \sum_{u=-\infty}^{\infty} g^{*}(u) g^{*}(u+m) e^{-j\left(2 \pi / N_{s s}\right) u n}$

Therefore, and similarly to (93)-(95), we obtain that

$$
\begin{aligned}
& {\left[\mathbf{G}_{\tau}^{*} \mathbf{G}_{\tau}^{H}\right]_{k+m, k}} \\
& =\frac{2 M+1}{N_{s s}} \sum_{n=-1}^{+1}\left[R_{g}^{\left(-1 / N_{s s}\right) n}(m)\right]^{*} e^{j\left(2 \pi / N_{s s}\right)\left(k-\tau_{c} / T_{s}\right) n} .
\end{aligned}
$$

\section{APPENDIX II}

\section{UML COST FUNCTION FOR NONOFFSET MODULATIONS}

In order to further expand the UML cost function in (32), it is convenient to decompose the outer-product matrix $\mathbf{G}_{\tau} \mathbf{G}_{\tau}^{H}$ only into those contributions that depend on the desired parameter $\tau$. In particular, we can see from (95) that the Fourier series expansion of the elements in $\mathbf{G}_{\tau} \mathbf{G}_{\tau}^{H}$ must be restricted to the cycle-frequencies $\alpha=\left\{-1 / N_{s s},+1 / N_{s s}\right\}$. The zero cyclefrequency can be avoided in the derivation as it does not depend on $\tau$

$$
\begin{aligned}
\mathbf{G}_{\tau} \mathbf{G}_{\tau}^{H} & \propto\left(e^{j(2 \pi / T) \tau_{c}} \boldsymbol{\Xi}_{g *}^{-1}+e^{-j(2 \pi / T) \tau_{c}} \boldsymbol{\Xi}_{g *}^{+1}\right) \\
{\left[\boldsymbol{\Xi}_{g *}^{n}\right]_{k+m, k} } & \doteq R_{g *}^{\left(1 / N_{s s}\right) n}(m) e^{j\left(2 \pi / N_{s s}\right) k n} .
\end{aligned}
$$

$$
\begin{aligned}
\psi\left(\frac{2 \pi}{N_{s s}} n ; m\right) & =\frac{1}{N_{s s}} \sum_{k=0}^{N_{s s}-1} \widetilde{R}_{g}(k ; m) e^{-j\left(2 \pi / N_{s s}\right) k n} \\
& =\frac{1}{N_{s s}} \sum_{k=0}^{N_{s s}-1} \sum_{n^{\prime}=-\infty}^{\infty} g^{*}\left(k-n^{\prime} N_{s s}\right) g\left(k-n^{\prime} N_{s s}+m\right) e^{-j\left(2 \pi / N_{s s}\right)\left(k+n^{\prime} N_{s s}\right) n}
\end{aligned}
$$


For an asymptotically large observation interval, we have that

$$
\begin{aligned}
\Lambda(\mathbf{r} \mid \tau) \propto & \operatorname{Tr}\left(\left[\left(\mathbf{G}_{\tau} \mathbf{G}_{\tau}^{H}\right) \odot \mathbf{V}\right] \widehat{\mathbf{R}}\right) \\
\propto & e^{j(2 \pi / T) \tau_{c}} \operatorname{Tr}\left(\left[\boldsymbol{\Xi}_{g *}^{-1} \odot \mathbf{V}\right] \widehat{\mathbf{R}}\right) \\
& +e^{-j(2 \pi / T) \tau_{c}} \operatorname{Tr}\left(\left[\boldsymbol{\Xi}_{g *}^{+1} \odot \mathbf{V}\right] \widehat{\mathbf{R}}\right)
\end{aligned}
$$

However, we can asymptotically expand the term $\operatorname{Tr}\left(\left[\boldsymbol{\Xi}_{g *}^{+1} \odot \mathbf{V}\right] \widehat{\mathbf{R}}\right)$ as follows:

$$
\begin{aligned}
& \operatorname{Tr}\left(\left[\boldsymbol{\Xi}_{g *}^{+1} \odot \mathbf{V}\right] \widehat{\mathbf{R}}\right) \\
& =\sum_{p=-M}^{M} \sum_{q=-M}^{M}\left[\boldsymbol{\Xi}_{g *}^{+1} \odot \mathbf{V}\right]_{p, q}[\widehat{\mathbf{R}}]_{q, p} \\
& =\left[\sum_{k=-M}^{M}\left[\boldsymbol{\Xi}_{g *}^{+1} \odot \mathbf{V}\right]_{k, k}[\widehat{\mathbf{R}}]_{k, k}\right. \\
& +\sum_{m=1}^{N-1} \sum_{k=-M}^{M-m}\left[\Xi_{g *}^{+1} \odot \mathbf{V}\right]_{k+m, k}[\widehat{\mathbf{R}}]_{k, k+m} \\
& \left.+\sum_{m=-1}^{-N+1} \sum_{k=-M+|m|}^{M}\left[\boldsymbol{\Xi}_{g *}^{+1} \odot \mathbf{V}\right]_{k+m, k}[\widehat{\mathbf{R}}]_{k, k+m}\right] \\
& =\left[\sum_{k=-M}^{M} R_{g *}^{1 / N_{s s}}(0) \Phi_{\bar{\omega}_{\epsilon}}(0) r(k) r^{*}(k) e^{j\left(2 \pi / N_{s s}\right) k}\right. \\
& +\sum_{m=1}^{N-1} R_{g *}^{1 / N_{s s}}(m) \Phi_{\bar{\omega}_{\epsilon}}(m) \sum_{k=-M}^{M-m} r(k) r^{*}(k+m) e^{j\left(2 \pi / N_{s s}\right) k} \\
& \left.+\sum_{m=-1}^{-N+1} R_{g *}^{1 / N_{s s}}(m) \Phi_{\bar{\omega}_{\epsilon}}(m) \sum_{k=-M+|m|}^{M} r(k) r^{*}(k+m) e^{j\left(2 \pi / N_{s s}\right) k}\right] \text {. }
\end{aligned}
$$

It is important to note that the range of the diagonal indexation $m$ in (100) and (104)-(105) depends on the effective time support of the pulse $g(k)$. Because $g(k)$ is a bandlimited pulse it has a theoretically infinite time support. However, most of its energy is concentrated on a finite interval which is in general negligible compared to $M$ when $M \rightarrow \infty$. For this reason, and without loss of generality, we can omit the diagonal indexation $m$ in the summation boundaries that incorporate $M$

$$
\begin{aligned}
& \lim _{M \rightarrow \infty} \sum_{k=-M}^{M-m} r(k) r^{*}(k+m) e^{j\left(2 \pi / N_{s s}\right) k} \\
& =\lim _{M \rightarrow \infty} \sum_{k=-M}^{M} r(k) r^{*}(k+m) e^{j\left(2 \pi / N_{s s}\right) k} \\
& \lim _{M \rightarrow \infty} \sum_{k=-M+|m|}^{M} r(k) r^{*}(k+m) e^{j\left(2 \pi / N_{s s}\right) k} \\
& =\lim _{M \rightarrow \infty} \sum_{k=-M}^{M} r(k) r^{*}(k+m) e^{j\left(2 \pi / N_{s s}\right) k} .
\end{aligned}
$$

Therefore, and for an asymptotic observation interval

$$
\begin{aligned}
\operatorname{Tr}\left(\left[\boldsymbol{\Xi}_{g *}^{+1} \odot \mathbf{V}\right] \widehat{\mathbf{R}}\right)= & \sum_{m=-M}^{M} R_{g *}^{1 / N_{s s}}(m) \Phi_{\bar{\omega}_{\epsilon}}(m) \\
& \cdot \sum_{k=-M}^{M} r(k) r^{*}(k+m) e^{j\left(2 \pi / N_{s s}\right) k}(108) \\
= & \sum_{m=-M}^{M} R_{g^{*}}^{1 / N_{s s}}(m) \Phi_{\bar{\omega}_{\epsilon}}(m) \\
& \cdot\left[\sum_{k=-M}^{M} r^{*}(k) r(k+m) e^{-j\left(2 \pi / N_{s s}\right) k}\right]^{*} \\
(M \rightarrow \infty) & \sum_{m=-M}^{M} R_{g *}^{1 / N_{s s}}(m) \Phi_{\bar{\omega}_{\epsilon}}(m)\left[R_{r *}^{1 / N_{s s}}(m)\right]^{*} .
\end{aligned}
$$

If we proceed in a similar way for the term $\operatorname{Tr}\left(\left[\boldsymbol{\Xi}_{g_{*}}^{-1} \odot \mathbf{V}\right] \widehat{\mathbf{R}}\right)$ and we apply the CAF property $R_{x}^{-\alpha}(m)=\left[R_{x}^{\alpha}(-m)\right]^{*} e^{-j 2 \pi \alpha m}$, then it is easy to show that we obtain the following:

$\operatorname{Tr}\left(\left[\Xi_{g *}^{-1} \odot \mathbf{V}\right] \widehat{\mathbf{R}}\right)=\sum_{m=-M}^{M}\left[R_{g *}^{1 / N_{s s}}(m)\right]^{*} \Phi_{\bar{\omega}_{\epsilon}}(m) R_{r *}^{1 / N_{s s}}(m)$

where we have used the fact that $\Phi_{\bar{\omega}_{\epsilon}}(-m)=\Phi_{\bar{\omega}_{\epsilon}}(m)$, according to the Toeplitz structure of $\mathbf{G}_{\tau} \mathbf{G}_{\tau}^{H}$. However, it is worth noting that (111) is the complex conjugate version of (110), as $\Phi_{\bar{\omega}_{\epsilon}}(m)$ is a real valued magnitude

$$
\operatorname{Tr}\left(\left[\Xi_{g *}^{+1} \odot \mathbf{V}\right] \widehat{\mathbf{R}}\right)=\left[\operatorname{Tr}\left(\left[\Xi_{g *}^{-1} \odot \mathbf{V}\right] \widehat{\mathbf{R}}\right)\right]^{*} .
$$

The complex conjugate equivalence in (112) is very important, and it allows us to express the asymptotic UML cost function in (102) as

\section{$\Lambda(\mathbf{r} \mid \tau)$}

$$
\begin{aligned}
& \propto e^{j(2 \pi / T) \tau_{c}} \operatorname{Tr}\left(\left[\Xi_{g *}^{-1} \odot \mathbf{V}\right] \widehat{\mathbf{R}}\right)+\left[e^{j(2 \pi / T) \tau_{c}} \operatorname{Tr}\left(\left[\boldsymbol{\Xi}_{g *}^{-1} \odot \mathbf{V}\right] \widehat{\mathbf{R}}\right)\right]^{*} \\
& \propto \operatorname{Re}\left[e^{j(2 \pi / T) \tau_{c}} \operatorname{Tr}\left(\left[\boldsymbol{\Xi}_{g *}^{-1} \odot \mathbf{V}\right] \widehat{\mathbf{R}}\right)\right] \\
& \propto \operatorname{Re}\left[e^{j(2 \pi / T) \tau_{c}} \sum_{m=-M}^{M} \Phi_{\bar{\omega}_{\epsilon}}(m)\left[R_{g *}^{1 / N_{s s}}(m)\right]^{*} R_{r *}^{1 / N_{s s}}(m)\right] .
\end{aligned}
$$

\section{APPENDIX III}

\section{UML COST FUNCTION FOR OFFSET MODULATIONS}

This Appendix is devoted to provide a detailed analysis on the derivation of the UML cost function for offset modulations in (71). Based on the cost function in (59)-(60), the objective of 
this Appendix is to relate the quadratic forms in (60) with their equivalent cyclostationary formulation in terms of the $\mathrm{CC}$ and NCC-CAF.

First of all, it is interesting to classify the quadratic forms in (60) into two different groups. On the one hand, the terms $\mathbf{r}^{H} \mathbf{G}_{\tau} \mathbf{G}_{\tau}^{H} \mathbf{r}$ and $\mathbf{r}^{H} \mathbf{J} \mathbf{G}_{\tau} \mathbf{G}_{\tau}^{H} \mathbf{J}^{T} \mathbf{r}$, and on the other hand, the terms $\mathbf{r}^{T} \mathbf{G}_{\tau}^{*} \mathbf{G}_{\tau}^{H} \mathbf{r}$ and $\mathbf{r}^{T} \mathbf{J} \mathbf{G}_{\tau}^{*} \mathbf{G}_{\tau}^{H} \mathbf{J}^{T} \mathbf{r}$. The first group is found to be related with the $\mathrm{CC}-\mathrm{CAF}$ whereas the second group is found to be related with the NCC-CAF.

To proof the above statement, let us start with the quadratic form $\mathbf{r}^{H} \mathbf{G}_{\tau} \mathbf{G}_{\tau}^{H} \mathbf{r}$. This term can be equivalently expressed as follows:

$$
\mathbf{r}^{H} \mathbf{G}_{\tau} \mathbf{G}_{\tau}^{H} \mathbf{r}=\operatorname{Tr}\left(\mathbf{G}_{\tau} \mathbf{G}_{\tau}^{H} \widehat{\mathbf{R}}\right)
$$

where $\widehat{\mathbf{R}} \doteq \mathbf{r r}^{H}$ is the sample covariance matrix of the received data. However, the right hand side of (116) is equivalent to the right hand side of (101) in Appendix II when $\mathbf{V}$ in (101) is an all-ones matrix. Therefore, we can benefit from the results in Appendix II for expressing $\mathbf{r}^{H} \mathbf{G}_{\tau} \mathbf{G}_{\tau}^{H} \mathbf{r}$ in terms of the cyclostationary formulation. From the mathematical derivation in (101)-(115) we have that

$$
\mathbf{r}^{H} \mathbf{G}_{\tau} \mathbf{G}_{\tau}^{H} \mathbf{r} \propto \operatorname{Re}\left[e^{j(2 \pi / T) \tau_{c}} \sum_{m=-M}^{M}\left[R_{g *}^{1 / N_{s s}}(m)\right]^{*} R_{r *}^{1 / N_{s s}}(m)\right]
$$

which coincides with (41) in Section IV-B, that is, the UML function for the timing estimation problem for nonoffset modulations in the absence of carrier-frequency error. From the mathematical derivations presented in Section IV-B and by means of the Parseval-Plancherel's theorem, it can be finally found that

$$
\mathbf{r}^{H} \mathbf{G}_{\tau} \mathbf{G}_{\tau}^{H} \mathbf{r} \propto \operatorname{Re}\left[e^{j(2 \pi / T) \tau_{c}} R_{y_{1} *}^{1 / N_{s s}}(0)\right]
$$

where $y_{1}(k)$ is the matched filter output of the received signal. A similar result can be assigned to the term $\mathbf{r}^{H} \mathbf{J G}_{\tau} \mathbf{G}_{\tau}^{H} \mathbf{J}^{T} \mathbf{r}$, as the matrix $\mathbf{J G}_{\tau}$ is just the matrix $\mathbf{G}_{\tau}$ with a $N_{s s} / 2$ time-shift delay. Hence, the result in (118) can be shifted accordingly by defining $y_{2}(k)$ as the $N_{s s} / 2$ time-shifted version of the signal $y_{1}(k)$. Thus, we have that

$$
\mathbf{r}^{H} \mathbf{J} \mathbf{G}_{\tau} \mathbf{G}_{\tau}^{H} \mathbf{J}^{T} \mathbf{r} \propto \operatorname{Re}\left[e^{j(2 \pi / T) \tau_{c}} R_{y_{2} *}^{1 / N_{s s}}(0)\right]
$$

At this point, (118) and (119) provide the equivalent cyclostationary formulation for the first group of quadratic forms in (60), that is, $\mathbf{r}^{H} \mathbf{G}_{\tau}^{*} \mathbf{G}_{\tau}^{H} \mathbf{r}$ and $\mathbf{r}^{H} \mathbf{J} \mathbf{G}_{\tau}^{*} \mathbf{G}_{\tau}^{H} \mathbf{J}^{T} \mathbf{r}$, respectively.

For the case of the second group of quadratic forms, that is, $\mathbf{r}^{H} \mathbf{G}_{\tau}^{*} \mathbf{G}_{\tau}^{H} \mathbf{r}$ and $\mathbf{r}^{H} \mathbf{J} \mathbf{G}_{\tau}^{*} \mathbf{G}_{\tau}^{H} \mathbf{J}^{T} \mathbf{r}$, an analysis similar to the one presented in Appendix II is required. Now, the difference is that we spart from the outer-product matrix $\mathbf{G}_{\tau}^{*} \mathbf{G}_{\tau}^{H}$ which can be expressed, similarly to (99), as follows:

$$
\begin{gathered}
\mathbf{G}_{\tau}^{*} \mathbf{G}_{\tau}^{H} \propto\left(e^{j(2 \pi / T) \tau_{c}}\left[\boldsymbol{\Xi}_{g}^{+1}\right]^{*}+e^{-j(2 \pi / T) \tau_{c}}\left[\boldsymbol{\Xi}_{g}^{-1}\right]^{*}\right) \\
{\left[\Xi_{g}^{n}\right]_{k+m, k} \doteq R_{g}^{\left(1 / N_{s s}\right) n}(m) e^{j\left(2 \pi / N_{s s}\right) k n} .}
\end{gathered}
$$

Let us start with the term $\mathbf{r}^{H} \mathbf{G}_{\tau}^{*} \mathbf{G}_{\tau}^{H} \mathbf{r}$ which can be expressed, similarly to (116) as

$$
\mathbf{r}^{H} \mathbf{G}_{\tau}^{*} \mathbf{G}_{\tau}^{H} \mathbf{r}=\operatorname{Tr}\left(\mathbf{G}_{\tau}^{*} \mathbf{G}_{\tau}^{H} \widetilde{\mathbf{R}}\right)
$$

where $\widetilde{\mathbf{R}}$ is given by $\widetilde{\mathbf{R}} \doteq \mathbf{r r}{ }^{T}$. By substituting (120) into (122), we have that

$$
\begin{aligned}
\mathbf{r}^{H} \mathbf{G}_{\tau}^{*} \mathbf{G}_{\tau}^{H} \mathbf{r}=e^{j(2 \pi / T) \tau_{c}} \operatorname{Tr}\left(\left[\boldsymbol{\Xi}_{g}^{+1}\right]^{*} \widetilde{\mathbf{R}}\right) \\
+e^{-j(2 \pi / T) \tau_{c}} \operatorname{Tr}\left(\left[\Xi_{g}^{-1}\right]^{*} \widetilde{\mathbf{R}}\right) .
\end{aligned}
$$

Contrary to (112) in Appendix II, and assuming $\mathbf{V}$ and all-ones matrix in (112), the two terms on the right hand side of (123) are not the complex conjugate one of each other. Therefore, there is no way to sum them together into a single expression. Following a similar mathematical derivation than the one presented in (103)-(110), it can be easily shown that

$$
\operatorname{Tr}\left(\left[\boldsymbol{\Xi}_{g}^{+1}\right]^{*} \widetilde{\mathbf{R}}\right)=\sum_{m=-M}^{M} R_{g}^{1 / N_{s s}}(m) R_{r}^{1 / N_{s s}}(m) .
$$

Therefore, the quadratic term under consideration results in

$$
\begin{aligned}
\mathbf{r}^{H} \mathbf{G}_{\tau}^{*} \mathbf{G}_{\tau}^{H} \mathbf{r}= & e^{j(2 \pi / T) \tau_{c}} \sum_{m=-M}^{M} R_{g}^{1 / N_{s s}}(m) R_{r}^{1 / N_{s s}}(m) \\
& +e^{-j(2 \pi / T) \tau_{c}} \sum_{m=-M}^{M} R_{g}^{-1 / N_{s s}}(m) R_{r}^{-1 / N_{s s}}(m) \\
= & e^{j(2 \pi / T) \tau_{c}} R_{y_{1}}^{1 / N_{s s}}(0)+e^{-j(2 \pi / T) \tau_{c}} R_{y_{1}}^{-1 / N_{s s}}(0)
\end{aligned}
$$

Regarding the remaining term $\mathbf{r}^{H} \mathbf{J} \mathbf{G}_{\tau}^{*} \mathbf{G}_{\tau}^{H} \mathbf{J}^{T} \mathbf{r}$, a similar result to (126) is obtained by using the $N_{s s} / 2$ time-shifted signal $y_{2}(k)$ instead of $y_{1}(k)$, that is

$$
\begin{aligned}
& \mathbf{r}^{H} \mathbf{J G}_{\tau}^{*} \mathbf{G}_{\tau}^{H} \mathbf{J}^{H} \mathbf{r} \\
& \quad=e^{j(2 \pi / T) \tau_{c}} R_{y_{2}}^{1 / N_{s s}}(0)+e^{-j(2 \pi / T) \tau_{c}} R_{y_{2}}^{-1 / N_{s s}}(0) .
\end{aligned}
$$

\section{REFERENCES}

[1] G. Vazquez and J. Riba, Signal Processing Advances in Wireless and Mobile Communications. Upper Saddle River, NJ: Prentice-Hall, 2000, vol. 2, Non-Data-Aided Digital Synchronization, ch. 9.

[2] J. Riba and G. Vazquez, "Bayesian recursive estimation of frequency and timing exploiting the cyclostationary property," Proc. EURASIP Signal Processing, vol. 40, no. 1, pp. 21-37, 1994.

[3] W. R. Benett, "Statistics of regenerative repeaters in digital transmission," Bell Syst. Tech. J., vol. 37, pp. 1501-1542, Nov. 1958.

[4] B. R. Saltzberg, "Timing recovery for synchronous binary data transmission,” Bell Syst. Tech. J., vol. 46, pp. 593-613, Mar. 1967.

[5] L. E. Franks and J. P. Bubrouski, "Statistical properties of timing jitter in a PAM timing recovery scheme," IEEE Trans. Commun., vol. 22, pp. 913-920, Jul. 1974.

[6] F. M. Gardner, "Self-noise in synchronizers," IEEE Trans. Commun., vol. 28, pp. 1159-1163, Aug. 1980.

[7] M. Moeneclaey, "Linear phase-locked loop theory for cyclostationary input disturbances," IEEE Trans. Commun., vol. 30, pp. 2253-2259, Oct. 1982. 
[8] A. N. D’Andrea, U. Mengali, and M. Moro, "Nearly optimum prefiltering in clock recovery," IEEE Trans. Commun., vol. 34, pp. 1081-1088, Nov. 1986.

[9] M. Oerder and H. Meyr, "Digital filter and square timing recovery," IEEE Trans. Commun., vol. 36, pp. 605-612, May 1988

[10] Y. Wang, E. Serpedin, and P. Ciblat, "Unified performance analysis of blind feedforward timing estimation," in Proc. 36th Asilomar Conference on Signals, Systems and Computers, vol. 2, Pacific Grove, CA, Nov. 3-6, 2002, pp. 1827-1831.

[11] S. A. Gromeneyer and A. L. McBride, "MSK and offset QPSK modulation," IEEE Trans. Commun., vol. 24, pp. 809-820, Aug. 1976.

[12] S. Pasupathy, "Minimum shift keying: A spectrally efficient modulation," IEEE Commun. Mag., vol. 17, no. 7, pp. 14-22, Jul. 1979.

[13] W. A. Gardner, Statistical Spectral Analysis. A Non Probabilistic Theory. Upper Saddle River, NJ: Prentice-Hall, 1988.

[14] - "Exploitation of spectral redundancy in cyclostationary signals," IEEE Signal Process. Mag., pp. 14-36, Apr. 1991.

[15] A. V. Dandawate and G. B. Giannakis, "Asymptotic theory of mixed time averages and kth-order cyclic moments and cimulant statistics," IEEE Trans. Inf. Theory, vol. 41, pp. 216-232, Jan. 1995.

[16] M. Moeneclaey and G. D. Jonghe, "Tracking performance comparison of two feedforward ML-oriented carrier-independent NDA symbol synchronizers," IEEE Trans. Commun., vol. 40, pp. 1423-1425, Sep. 1992.

[17] U. Mengali and A. N. D'Andrea, Synchronization Techniques for Digital Receivers. New York: Plenum, 1997

[18] J. A. Lopez-Salcedo and G. Vazquez, "Stochastic approach to square timing estimation with frequency uncertainty," in Proc. IEEE International Conference on Communications (ICC'03), vol. 5, Anchorage, AK, May 2003, pp. 3555-3559.

[19] A. Papoulis, Probability, Random Variables and Stochastic Processes. New York: McGraw-Hill, 1991.

[20] Y. Wang, E. Serpedin, and P. Ciblat, "Blind feedforward cyclostationaybased timing estimation for linear modulations," IEEE Trans. Wireless Commun., vol. 3, pp. 709-715, May 2003.

[21] E. W. Weisstein, CRC Concise Encyclopedia of Mathematics. Boca Raton, FL: Chapman and Hall/CRC, 1999.

[22] F. Gini and G. B. Giannakis, "Frequency offset and symbol timing recovery in flat-fading channels: A cyclostationary approach," IEEE Trans. Commun., vol. 46, pp. 400-411, Mar. 1998.

[23] A. V. Oppenheim and R. W. Schafer, Discrete-Time Signal Processing. Englewood Cliffs, NJ: Prentice-Hall, 1989.

[24] Y. Wang, P. Ciblat, E. Serpedin, and P. Loubaton, "Performance analysis of a class of nondata aided frequency offset and symbol timing estimators for flat-fading channels," IEEE Trans. Signal Process., vol. 50, pp. 2295-2305, Sep. 2002.

[25] A. Viterbi, "Nonlinear estimation of PSK-modulated carrier phase with application to burst digital transmission," IEEE Trans. Inf. Theory, vol. 29, pp. 543-551, Jul. 1983.
[26] R. D. Gitlin, J. F. Hayes, and S. B. Weinstein, Data Communications Principles. New York: Plenum, 1992.

[27] A. A. D'Amico, A. N. D'Andrea, and U. Mengali, "Feedforward joint phase and timing estimation with OQPSK modulation," IEEE Trans Veh. Technol., vol. 48, pp. 824-832, May 1999.

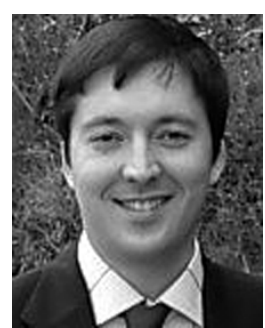

José A. López-Salcedo (S'98) was born in Barcelona, Catalonia, Spain, in 1978. He received the M.S. degree in telecommunication engineering and the advanced studies diploma in signal theory and communications both from the Technical University of Catalonia (UPC), Barcelona, in 2001 and 2004, respectively. The same year, he joined the Department of Signal Theory and Communications at UPC, where he is currently working toward the Ph.D. degree. Since 2002, he holds a grant from the Spanish Ministry of Education and Science in support of his $\mathrm{Ph} . \mathrm{D}$. studies.

His research interests are in statistical signal processing, information theory and digital communications with special attention to ultra-wideband (UWB) systems.

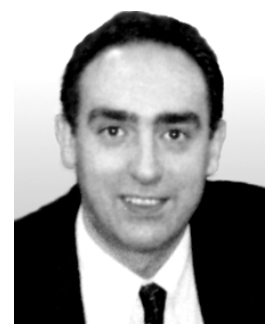

Gregori Vázquez (SM'98) was born in Barcelona, Spain, in 1961. He received the M.S. and Ph.D. degrees in telecommunications engineering from the Technical University of Catalonia (UPC), Barcelona, in 1984 and 1988, respectively.

$\mathrm{He}$ is a Professor at the Department of Signal Theory and Communications of the Technical University of Catalonia. His general interests are statistical signal processing and digital communications.

Dr. Vázquez is a recipient of the 2003 Best Paper Award of the IEEE Signal Processing Society. He is serving as Associate Editor of the IEEE TRANSACTIONS ON SIGNAL PROCESSING since 1999, and is also serving as a Member of the Editorial Board of the IEEE SIGNAL PROCESSING MAGAZINE and as a Member the Signal Processing for Communications Technical Committee, as well. He is the Chairman of the Research Program on Communications of the Spanish Science and Technology Ministry. 